UNIVERSITY OF TECHNOLOGY SYDNEY

Centre for Quantum Software and Information

\title{
Decomposition of Quantum Markov Chains and Its Applications
}

\author{
by \\ Ji Guan \\ A Thesis Submitted
in Partial Fulfillment of the
Requirements For the Degree
}

Doctor of Philosophy

Sydney, Australia 


\section{Certificate of Authorship/Originality}

I certify that the work in this thesis has not been previously submitted for a degree nor has it been submitted as a part of the requirements for other degrees except as fully acknowledged within the text.

I also certify that this thesis has been written by me. Any help that I have received in my research and in the preparation of the thesis itself has been fully acknowledged. In addition, I certify that all information sources and literature used are quoted in the thesis.

(C) Copyright 2018 Ji Guan 


\section{ABSTRACT \\ Decomposition of Quantum Markov Chains and Its Applications \\ by \\ Ji Guan}

Markov chains have been widely employed as a fundamental model in the studies of probabilistic and stochastic communicating and concurrent systems. It is wellunderstood that decomposition techniques play a crucial role in reachability analysis and model-checking of Markov chains. (Discrete-time) Quantum Markov chains have been introduced as a model of quantum communicating systems [66] and also a semantic model of quantum programs [67]. The BSCC (Bottom Strongly Connected Component) and stationary coherence decompositions of quantum Markov chains were introduced in [62, 68, 5]. This thesis presents a new decomposition technique, namely periodic decomposition, for quantum Markov chains. This decomposition further helps us find sufficient and necessary conditions for limiting states of quantum Markov chains.

To confirm the power of these decomposition techniques, we apply them to characterizing the one-shot zero-error capacity of quantum channels, finding the structure of quantum decoherence-free subsystems against quantum noises and superactivating quantum memory with entanglement via modeling the underlying quantum systems by quantum Markov chains.

Dissertation directed by Professor Mingsheng Ying and Professor Yuan Feng

Centre for Quantum Software and Information

Faculty of Engineering and Information Technology 


\section{Acknowledgements}

I am thankful to many people for their help and support during my work on this thesis. Below, I would like to acknowledge those who participated in my supervision, research, and everyday life.

First of all, I would like to thank my direct supervisors Prof. Mingsheng Ying and Prof. Yuan Feng for guiding me through the not always serene waters of research. Without their care, encouragement and steering this thesis would never be written. Next, I thank all colleagues who contributed to the presented work in many different ways, such as joint papers, valuable discussions and finding serious flaws in early versions of my work.

Last but not least, I would like to thank my family. My beloved father and mother, who gave me life and made me who I am. My little sister of whom I am proud. Thank you all for being in my heart.

Ji Guan

Sydney, Australia, 2018. 


\section{List of Publications}

\section{Journal Papers}

J-1. Guan, J., Feng, Y. and Ying, M., 2018. Decomposition of quantum Markov chains and its applications. Journal of Computer and System Sciences, 95, pp.55-68.

J-2. Su, Z., Guan, J. and Li, L., 2018. Efficient quantum repeater with respect to both entanglement-concentration rate and complexity of local operations and classical communication. Physical Review A, 97(1), p.012325.

J-3. Liu, S., Zhou, L., Guan, J., He, Y., Duan, R. and Ying, M., 2017. Q $|S I\rangle$ : a quantum programming environment. SCIENTIA SINICA Informationis 47(10), 1300-1315(2017);

\section{Preprint}

P-1. Guan, J., Feng, Y. and Ying, M., 2017. Super-activating Quantum Memory with Entanglement. arXiv preprint arXiv:1708.00700.

P-2. Guan, J., Feng, Y. and Ying, M., 2018. The structure of decoherence-free subsystems, arXiv preprint arXiv:1802.04904, 2018.

P-3. Liu, S., Wang, X., Zhou, L., Guan, J., Li, Y., He, Y., Duan, R. and Ying, M., 2017. $Q|S I\rangle$ : a quantum programming environment. arXiv preprint arXiv:1710.09500. 


\section{Contents}

Certificate ii

Abstract iii

Acknowledgments

List of Publications $\quad \mathrm{V}$

Abbreviation ix

\begin{tabular}{lll}
\hline Introduction & 1
\end{tabular}

\begin{tabular}{llr}
\hline II Preliminaries & 7
\end{tabular}

1 Review of Classical Markov Chains 9

1.1 Markov Chains . . . . . . . . . . . . . . . . . . . . . . 9

1.2 Classification of States and Chains . . . . . . . . . . . . . . . . 11

1.3 Stationary Distribution and Limit Theorem . . . . . . . . . . . . . . . 12

2 Review of Quantum Markov Chains 14

2.1 Definition . . . . . . . . . . . . . . . . . . . . . . . . . . . . . . . . . . 14

2.2 Essential Tools . . . . . . . . . . . . . . . . . . . . . . . . . . . . . . . . . . . . . . 15

III Decomposition of Quantum Markov Chains 18

3 BSCC Decomposition and Reachability 20

3.1 Reachability . . . . . . . . . . . . . . . . . . . . . 20 
3.2 Classification of Quantum States . . . . . . . . . . . . . . . . 21

3.3 BSCC Decomposition . . . . . . . . . . . . . . . . . . . . . . . 23

4 Stationary Coherence Decomposition and Fixed Points 24

4.1 Stationary Coherence Decomposition . . . . . . . . . . . . . . . . . . . 24

4.2 Fixed Points . . . . . . . . . . . . . . . . . . . . . . . 26

5 Periodic Decomposition and Limiting States 30

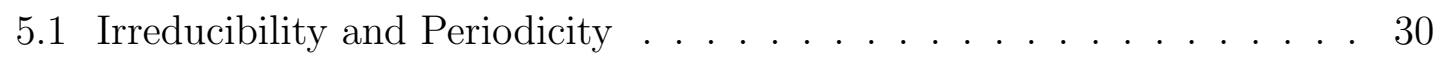

5.2 Periodic Decomposition $\ldots \ldots \ldots$. . . . . . . . . . . . . . . . 39

5.3 Limiting States . . . . . . . . . . . . . . . . . . . . . 4 41

5.4 Conclusion $\ldots \ldots \ldots \ldots$. . . . . . . . . . . . . . . . . . . . . 42

\begin{tabular}{lll}
\hline IV Applications & 44
\end{tabular}

6 One-shot Zero-error Capacity of Quantum Channels 46

6.1 One-shot Zero-error Capacity . . . . . . . . . . . . . . . . . . . . 46

6.2 A New Characterization . . . . . . . . . . . . . . . . . 51

6.3 Conclusion . . . . . . . . . . . . . . . . . . . . . . . . . . 52

7 The Structure of Decoherence-free Subsystems 54

7.1 Continuous Coherence . . . . . . . . . . . . . . . . . . . . . . . . . 55

7.2 Structure Theorem . . . . . . . . . . . . . . . . . . . . 58

7.3 Matrix Product States . . . . . . . . . . . . . . . . . . . . 65

7.4 Conclusion . . . . . . . . . . . . . . . . . . . . . . . 67

8 Super-activating Quantum Memory with Entanglement 68

8.1 Quantum Memory . . . . . . . . . . . . . . . . . . . . . . . . . . . 69 
8.2 Super-activation for Storing Classical Information . . . . . . . . . . . . 70

8.3 Super-activation for Storing Quantum Information . . . . . . . . . . . 73

8.4 Conclusion . . . . . . . . . . . . . . . . . . . . . . . . . . . 77



\begin{tabular}{llr}
\hline VI & Appendix & 80
\end{tabular}

$\begin{array}{ll}\text { Bibliography } & 83\end{array}$ 


\section{Abbreviation}

MC - Markov chain

qMC - quantum Markov chain

gcd - the greatest common divisor

BSCC - bottom strongly connected component

CPTP - completely positive and trace-preserving

RHS - right-hand side

LHS - left-hand side 
Part I

Introduction 


\section{Classical Markov Chains}

A Markov chain (MC), named after the Russian mathematician Andrey Markov ( June 14, 1856-July 20, 1922), is a discrete-time Markov process with finite states [27]. A Markov process is a stochastic process in which the current state is sufficient to predict the future state (i.e. the state after the current state), while the past (i.e. the previous history of the current state) is irrelevant. It can be regarded as a stepby-step process. At each step, a Markov chain can jump from one state to another state or maintain the current state. We say that the changes of states of the chain are transitions. The probabilities associated with state changes are called transition probabilities. In summary, a Markov chain is characterized by a finite state space and a transition probability matrix consisting of all transition probabilities.

In 1906 Markov provided the basic definition of what was later to be called a Markov chain. Kolmogorov gave a generalization to countably infinitely state spaces in 1936. Markov chains are related to two important topics of physics in the early twentieth century, namely Brownian motion and ergodic hypothesis, but Markov appeared to have pursued this out of the extension of the law of large numbers to dependent events. Then, he applied his findings for the first time to the first 20,000 letters of Pushkin's Eugene Onegin in 1913. See [23] for more details.

Markov chains have many applications modeling practical systems in a wide range of topics such as chemistry [39], information sciences [52], economics [28], social sciences [1] and music [46]. Specifically, in the field of Internet, the PageRank of a website used by Google is defined by a Markov chain to order search results [50]. The PageRank of a page $i$ is the probability to be at $i$ in the stationary distribution of the Markov chain on all pages of the results. Also, Markov chains have been used to analyze web navigation behavior of users. They can model a user's web link transition on a particular website and make forecasts regarding future exploration 
and to personalize the web page for him or her. Furthermore, some researchers have extensively employed Markov chains as a fundamental model of probabilistic and stochastic communicating and concurrent systems. In particular, various algorithmic analysis and model checking techniques have been developed for them in the last three decades (see for example [3], Chapter 10).

\section{Quantum Markov Chains}

A quantum Markov chain (qMC) is a quantum generalization of a Markov chain, where the state space is a Hilbert space and the transition probability matrix is replaced by a super-operator which is a mathematical formalization of the discretetime evolution of (open) quantum systems. Quantum Markov chains provide a potential paradigm for modeling the development of quantum systems.

Continuous-time quantum Markov processes have been intensively studied in mathematical physics for many years, and achieved several discoveries of fundamental importance [18, 19, 44, 22]. Recently, they also have been applied to quantum control setting, such as stabilizing quantum systems [60, 30, 59] and preparing quantum states [61, 54, 58]. Discrete-time quantum Markov chains were introduced in [67, 70, 68, as a semantic model targeting verification and termination analysis of quantum programs.

A particular class of quantum Markov chains in closed systems, called quantum walks, has been successfully employed in the design of quantum algorithms (see [2, 31] for a survey of this line of research). In open quantum systems (noises allowed), a similar model, called open quantum random walks, has been introduced, on lattices as well as on finite graphs [11].

This thesis focuses on the decompositions of (discrete-time) quantum Markov chains and targets applications to quantum information theory, especially informa- 
tion preserving. We start with introducing existing decompositions of quantum Markov chains, namely the BSCC and stationary coherence decompositions. Then we add a new decomposition technique, called periodic decomposition. Furthermore, the characterization of limiting states of quantum Markov chains has been found. More importantly, all these mathematical tools have been applied in quantum information theory as follows.

Zero-error capacity is defined as the least upper bound of the rates at which one can send classical messages correctly via a quantum channel. The periodic decomposition of quantum Markov chains together with the BSCC decomposition provides us with a new angle to look at the structure of quantum channels. In particular, it gives a new characterization of one-shot zero-error capacity for general quantum channels.

Decoherence-free subsystems have been successfully formed as a tool to preserve fragile quantum information against noises. With the help of the BSCC and stationary coherence decompositions, we develop a structure theory for decoherence-free subsystems. Based on it, we present an effective algorithm to construct a set of maximal decoherence-free subsystems in the sense that any other such subsystem is a subspace of one of them. Applying these techniques in quantum many-body systems, we propose a simple and numerically robust method to determine if two irreducible tensors are repeated, an essential step in deciding if they are equivalent in generating matrix product states.

As a particular case of decoherence-free subsystems, noiseless subsystems can directly protect quantum states without any correction. Because of this property, they were employed to design a general (hybrid) quantum memory cell model that can store both quantum and classical information. Applying the stationary coherence and periodic decomposition techniques, we find an interesting new phenomenon that 
a pair of purely classical memory cells (cannot store quantum information alone) can be used to preserve quantum states, whereas two null memory cells (quantum and classical information both cannot be stored in each of them) can only be employed together to encode classical information. These findings reveal the superactivation of memory cells. Furthermore, necessary and sufficient conditions for this phenomenon are discovered so that the super-activation can be simply checked by examining certain eigenvalues of the quantum memory cell without computing the noiseless subsystems explicitly. In particular, it is found that entangled and separable stationary states are responsible for the super-activation of storing quantum and classical information, respectively.

\section{Thesis Organization}

This thesis is organized as follows:

Part II: Preliminaries

- Chapter 1 We review some basic notions and properties of classical Markov chains. This will help readers understand the latter generalization to the quantum world.

- Chapter 2. We show how to generalize classical Markov chains to the quantum case and provide some useful tools in the analysis of quantum Markov chains, such as eigenvalues and matrix representations.

Part III Decomposition of Quantum Markov Chains

- Chapter 3: The reachability of quantum Markov chains will be given. It further deduces the BSCC decomposition developed in [68, 62].

- Chapter 4: This chapter presents the stationary coherence decomposition of 
quantum Markov chains from [5]. Such decomposition reveals the structure of fixed points of quantum Markov chains.

- Chapter 5: We extend the notions of irreducibility and periodicity for classical Markov chains to quantum Markov chains, and show that they coincide with the corresponding notions presented in the literature from different perspectives. We then carefully examine limiting states of irreducible and aperiodic quantum Markov chains, and a periodic decomposition of irreducible quantum Markov chains is presented.

Part IV] Applications

- Chapter 6; A new characterization of one-shot zero-error capacity of quantum channels will be given in terms of the BSCC and periodic decompositions.

- Chapter 75: We obtain the structure of decoherence-free subsystems by the BSCC and stationary coherence decompositions.

- Chapter 8: Applying the stationary coherence and periodic decompositions, we show that entanglement can super-activate quantum memory.

Part V: Concluding Remarks

A summary of the thesis contents and its contributions are given in the final part. Recommendation for future works is given as well. 
Part II

\section{Preliminaries}


In this part, we will first review basic definitions of classical Markov chains and present some important results of classical Markov chains. Then we move to the quantum case by introducing quantum Markov chains. Meanwhile, we list some useful tools from previous literature for our later use. 


\section{Chapter 1}

\section{Review of Classical Markov Chains}

Classical Markov chains (simply referred as Markov chains) have been intensely and completely studied in the last century. In this chapter, we are going to review the basic notions and properties of Markov chains, which helps the reader to understand the generalization to the quantum world in the next chapter. We start by presenting the definition of Markov chains in Section 1.1. Then different kinds of states and chains are shown in Section 1.2. In the last section, we will discuss stationary and limiting distributions of Markov chains.

\subsection{Markov Chains}

In brief, a Markov chain is a random process with the property that, conditional on its current value, the future is independent of the past. Formally,

Definition 1: A Markov chain is a discrete-time stochastic process $\left(X_{n}, n \geq 0\right)$ such that each random variable $X_{n}$ takes values in a discrete and finite set $S$ (the state space) and satisfies

$$
P\left(X_{n+1}=j \mid X_{n}=i, X_{n-1}=i_{n-1}, \cdots, X_{0}=i_{0}\right)=P\left(X_{n+1}=j \mid X_{n}=i\right)
$$

for all $n \geq 0$ and all states $i, j, i_{0}, \cdots, i_{n-1} \in S$.

The evolution of a chain is described by its transition probabilities, $P\left(X_{n+1}=\right.$ $\left.j \mid X_{n}=i\right)$; it can be quite complicated in general since these probabilities depend on three arguments $n, i$ and $j$. We shall restrict our attention to the case when they do not depend on $n$ but only upon $i$ and $j$. 
Definition 2: The chain $\left(X_{n}, n \geq 0\right)$ is called homogeneous if

$$
P\left(X_{n+1}=j \mid X_{n}=i\right)=P\left(X_{1}=j \mid X_{0}=i\right)
$$

for all $n, i, j$. The transition matrix $P=\left(p_{i j}\right)$ is the $|S| \times|S|$ matrix of transition probabilities

$$
p_{i j}=P\left(X_{n+1}=j \mid X_{n}=i\right) .
$$

Henceforth, all classical Markov chains are assumed homogeneous. Therefore, a Markov chain can be simply formed as a tuple $(S, P)$, where $S$ is a finite state set and $P$ is a transition matrix over $S$.

Definition 3: The $n$-step transition probability is $p_{i j}^{(n)}=P\left(X_{n}=j \mid X_{0}=i\right)$ for all $i, j \in S$.

The adjacency relation between states is important to the analysis of Markov chains, so we introduce as follows.

Definition 4: Given a Markov chain $(S, P)$ and any states $i, j \in S$, we say that $j$ is adjacent to $i$, written $i \rightarrow j$, if $p_{i j}>0$. Furthermore, $j$ is reachable from $i$ if there exists a $n \geq 0$ such that $p_{i j}^{(n)}>0$.

Definition 5: Given a Markov chain $(S, P)$, for two states $i$ and $j$, we say that they communicate if $i$ is reachable from $j$ and $j$ is reachable from $i$.

Now, let us group states together according to reachability.

Definition 6: A class $\mathcal{C}$ of states is a non-empty set of states such that

- For any two distinct states in $\mathcal{C}$, they communicate;

- Any state $i \in \mathcal{C}$ cannot communicate with any state $j \notin \mathcal{C}$. 


\subsection{Classification of States and Chains}

In this section, we give different types of states and show a Markov chain can be divided into disjoint subsets in which the states belong to the same type.

Firstly, sometimes we shall be interested in the epochs of time at which return to the starting state is possible.

Definition \%: For any state $i$, let $d(i)=\operatorname{gcd}\left\{n \geq 1: p_{i i}^{(n)}>0\right\}$, where ged stands for the greatest common divisor.

- If $p_{i i}^{(n)}=0$ for all $n \geq 1$, then we say state $i$ has no period or it has period 0 ;

- If $d(i)=1$, then we say state $i$ is aperiodic or it has period 1 ;

- If $d(i) \geq 2$, then we say state $i$ is periodic with period $d(i)$.

When all states of a Markov chain have the same period $d>0$, we say that the chain is $d$-periodic if $d \geq 2$ or aperiodic if $d=1$.

If a Markov chain is $d$-periodic, it means that the chain starting from a state $i$ cannot return to $i$ at these time epochs that have no the divisor $d$. In the above definition, we have defined the period of a Markov chain, and another relevant question is whether or not the chain starting from state $i$ will eventually come back to $i$. With this in mind, we make the following definition.

Definition 8: A state $i \in S$ is recurrent if it is reachable from all states that are reachable from $i$. A transient state is a state that is not recurrent. When all states are recurrent (transient), we say that the chain is recurrent (transient).

Lemma 1: For a Markov chain, either all states in a class are transient or all are recurrent. Furthermore, all states in the same class have the same period.

From the above lemma, we can directly say that a class is recurrent or transient with the period of its states. We can also observe that any state cannot be in two 
different classes at the same time. So we can partition the state space into several classes:

Theorem 1: For a Markov chain $(S, P)$, the state space can be uniquely partitioned into finite classes.

If a Markov chain has only one class, then the chain is called an irreducible Markov chain; formally

Definition 9: A Markov chain $(S, P)$ is irreducible if $S$ is a class.

In an irreducible Markov chain, there is always a path from states $i$ to $j$ if $i \neq j$. All states in an irreducible Markov chain are recurrent.

\subsection{Stationary Distribution and Limit Theorem}

Stationary and limiting distributions play essential roles in stabilizing Markov chains. This section presents the characterizations of both regarding irreducibility and periodicity.

Definition 10: A distribution $\pi^{*}$ is called stationary if it satisfies the equation

$$
\pi^{*}=\pi^{*} P
$$

where $\pi^{*} P$ is the product of $\pi^{*}$ (vector) and $P$ (matrix).

It is easy to see that any Markov chain admits at least one stationary distribution. In particular, an irreducible Markov chain has only one stationary distribution.

Lemma 2: For a Markov chain $(S, P)$, it is irreducible if and only if it has only one stationary distribution and the distribution is faithful, i.e. all elements are non-zero.

The following theorem discusses when the chain has the stationary distribution as the limiting one. 
Theorem 2: An irreducible and aperiodic Markov chain $(S, P)$ has a unique stationary distribution $\pi^{*}$. This distribution is also a limiting distribution in the sense

$$
\lim _{n \rightarrow \infty} \pi_{i}^{(n)}=\pi_{i}^{*}, \text { for all } i \in S \text { and } \pi^{(0)}
$$

where $\pi^{(0)}$ is the initial distribution and $\pi^{(n)}=\pi^{(0)} P^{n}$ at any finite time $n$. 


\section{Chapter 2}

\section{Review of Quantum Markov Chains}

In this chapter, we review some basic notions and results of quantum Markov chains; for details we refer to [49].

\subsection{Definition}

Recall that a (classical) Markov chain is a random process in which the future behavior depends only on the present, and a matrix of transition probabilities models the evolution of such a process. Note that the evolution of an open quantum system can be modeled mathematically by a super-operator, i.e., a completely positive and trace-preserving (CPTP) linear map, acting on its state Hilbert space of the system. This naturally motivates us to present the following:

Definition 11 ([68]): A quantum Markov chain is a pair $(\mathcal{H}, \mathcal{E})$, where $\mathcal{H}$ is a Hilbert space, and $\mathcal{E}$ is a super-operator on $\mathcal{H}$.

In this thesis, we only consider finite-dimensional quantum Markov chains, i.e. $\operatorname{dim}(\mathcal{H})<\infty$. For any two Hilbert spaces $\mathcal{H}$ and $\mathcal{H}^{\prime}$, let $\mathcal{L}\left(\mathcal{H}, \mathcal{H}^{\prime}\right)$ be the set of all linear operators from $\mathcal{H}$ to $\mathcal{H}^{\prime}$. Simply, we also let $\mathcal{L}(\mathcal{H})=\mathcal{L}(\mathcal{H}, \mathcal{H})$ and $D(\mathcal{H})$ denote the set of density operators in $\mathcal{H}$. Then a state of a quantum Markov chain $(\mathcal{H}, \mathcal{E})$ is an operator $\rho \in D(\mathcal{H})$. Recall that the support of a density operator $\rho$, denoted $\operatorname{supp}(\rho)$, is the subspace of $\mathcal{H}$ linearly spanned by the eigenvectors of $\rho$ corresponding to non-zero eigenvalues. The image of a subspace $\mathcal{X}$ of $\mathcal{H}$ under a 
super-operator $\mathcal{E}$ is defined to be

$$
\mathcal{E}(\mathcal{X}):=\bigvee_{|\phi\rangle \in \mathcal{X}} \operatorname{supp}(\mathcal{E}(|\phi\rangle\langle\phi|))
$$

Here $|\phi\rangle$ denotes a pure state in $\mathcal{X}$. The join of a family $\left\{\mathcal{X}_{k}\right\}$ of subspaces of $\mathcal{H}$ is defined by $\bigvee_{k} \mathcal{X}_{k}=\operatorname{lin} . \operatorname{span}\left(\bigcup_{k} \mathcal{X}_{k}\right)$, and for a set of vectors $\mathcal{Y}, \operatorname{lin} \cdot \operatorname{span}(\mathcal{Y})=$ $\left\{\sum_{i=1}^{k} \lambda_{i} v_{i} \mid k \in \mathbb{N}, v_{i} \in \mathcal{Y}, \lambda_{i} \in \mathbb{C}\right\}$ is the space spanned by vectors in $\mathcal{X}$.

For any linear map $\mathcal{E}$ on $\mathcal{L}(\mathcal{H})$, if $\operatorname{dim}(\mathcal{H})=n$, then it admits up to $n^{2}$ distinct (complex) eigenvalues $a$ satisfying

$$
\mathcal{E}(A)=a A
$$

for some $A \in \mathcal{L}(\mathcal{H}), A \neq 0$. We write $\lambda(\mathcal{E})$ for the set of all eigenvalues of $\mathcal{E}$. The spectral radius of $\mathcal{E}$ is defined as $\varrho(\mathcal{E}):=\sup \{|\lambda|: \lambda \in \operatorname{spec}(\mathcal{E})\}$. In particular, if $\mathcal{E}$ is a CPTP map, then $\varrho(\mathcal{E})=1$.

Definition 12 ([68]): For a quantum Markov chain $\mathcal{G}=(\mathcal{H}, \mathcal{E})$, a state $\rho \in D(\mathcal{H})$ is called stationary if $\mathcal{E}(\rho)=\rho$; that is, $\rho$ is a fixed point of $\mathcal{E}$. Furthermore, $\rho$ is said to be minimal if there is no stationary state $\sigma$ such that $\operatorname{supp}(\sigma) \subsetneq \operatorname{supp}(\rho)$.

We collect all fixed points of $\mathcal{E}$ as $f i x(\mathcal{E})=\{X \in \mathcal{L}(\mathcal{H}) \mid \mathcal{E}(X)=X\}$.

\subsection{Essential Tools}

In this section, we present some useful tools in analysis of quantum Markov chains, which will be frequently used in the latter discussion.

Let $\mathcal{E}$ be a super-operator with Kraus operators $\left\{E_{i}\right\}$, i.e. $\mathcal{E}(\cdot)=\sum_{i} E_{i} \cdot E_{i}^{\dagger}$. Then its matrix representation [67] is defined to be $M=\sum_{i} E_{i} \otimes E_{i}^{*}$ where $E^{*}$ stands for the (entry-wise) complex conjugate of $E$. Assume that $M=S J S^{-1}$ is the Jordan decomposition of $M$, where

$$
J=\sum_{k=1}^{K} \lambda_{k} P_{k}+N_{k},
$$


$N_{k}^{d_{k}}=0$ for some $d_{k}>0, N_{k} P_{k}=P_{k} N_{k}=N_{k}, P_{k} P_{l}=\delta_{k l} P_{k}, \operatorname{tr}\left(P_{k}\right)=d_{k}$, and $\sum_{k} P_{k}=I$. Let

$$
\begin{aligned}
J_{\infty} & :=\sum_{k: \lambda_{k}=1} P_{k}, \\
J_{\phi} & :=\sum_{k:\left|\lambda_{k}\right|=1} P_{k} .
\end{aligned}
$$

Then we write:

- $\mathcal{E}_{\infty}$ for the super-operator with the matrix representation $S J_{\infty} S^{-1}$.

- $\mathcal{E}_{\phi}$ for the super-operator with the matrix representation $S J_{\phi} S^{-1}$.

$\mathcal{E}_{\infty}$ and $\mathcal{E}_{\phi}$ admit the following interesting characterizations.

Lemma 3 ([66, Proposition 6.3]): For any quantum Markov chain $\mathcal{G}=(\mathcal{H}, \mathcal{E})$,

(1) there exists an increasing sequence of integers $n_{i}$ such that $\mathcal{E}_{\phi}=\lim _{i \rightarrow \infty} \mathcal{E}^{n_{i}}$;

(2) $\mathcal{E}_{\infty}=\lim _{N \rightarrow \infty} \frac{1}{N} \sum_{n=1}^{N} \mathcal{E}^{n}$

Meanwhile, we collect some other results in the previous literature for later use.

Lemma 4 ([70]): For any quantum Markov chain $\mathcal{G}=(\mathcal{H}, \mathcal{E})$, real number $p>0$, $\rho \in D(\mathcal{H})$, and $\mathcal{X}, \mathcal{Y}$ being subspaces of $\mathcal{H}$, we have

(1) $\operatorname{supp}(p \rho)=\operatorname{supp}(\rho)$;

(2) $\mathcal{E}(\operatorname{supp}(\rho))=\operatorname{supp}(\mathcal{E}(\rho))$

(3) if $\mathcal{X} \subseteq \mathcal{Y}$, then $\mathcal{E}(\mathcal{X}) \subseteq \mathcal{E}(\mathcal{Y})$;

(4) $\mathcal{E}(\mathcal{X} \bigvee \mathcal{Y})=\mathcal{E}(\mathcal{X}) \bigvee \mathcal{E}(\mathcal{Y})$

Lemma 5 ([51] ): If $\mathcal{F}$ is a CP map with $\mathcal{F}(\cdot)=\sum_{i} F_{i} \cdot F_{i}^{\dagger}$ and $\sum_{i} F_{i}^{\dagger} F_{i} \leq I$, then for any Hermitian matrix $A$,

$$
\|\mathcal{F}(A)\|_{1} \leq\|A\|_{1}
$$


Lemma 6 ([29]): Let $S(\mathcal{H})$ denote the set of all subspaces of $\mathcal{H}$. Then for any $\rho \in D(\mathcal{H})$

$$
\inf _{\mathcal{X} \in S(\mathcal{H}) \backslash\{\mathbf{0}\}} \operatorname{tr}\left(P_{\mathcal{X}} \rho\right)=\lambda_{\min }(\rho)
$$

where $\mathbf{0}$ is the zero-dimensional subspace and $\lambda_{\min }(\rho)$ is the minimum eigenvalue of $\rho$. 


\title{
Part III
}

\section{Decomposition of Quantum}

\author{
Markov Chains
}


In this part, the three-level decomposition of quantum Markov chains will be given, namely the BSCC, stationary coherence and periodic decompositions. They have strong connections with the essential properties of quantum Markov chains: reachability, fixed points and limiting states, respectively. 


\section{Chapter 3}

\section{BSCC Decomposition and Reachability}

Reachability analysis plays an essential role in verification and model-checking of both classical and probabilistic systems. In [55], the reachability of quantum systems was firstly studied by physicists. And then reachability of quantum Markov chains was considered by [70] and it was used in termination checking of concurrent quantum programs. Recently, [68] studied three kinds of long-term behaviors, namely reachability, repeated reachability and persistence of quantum Markov chains by introducing the notion of bottom strongly connected component(BSCC) and the related decomposition of quantum Markov chains. Meanwhile, they designed algorithms for computing the reachability, repeated reachability and persistence probabilities of a quantum Markov chain and analyzed their complexities.

In this chapter, we first introduce the reachability of quantum Markov chains in Section 3.1, which defines adjacency relation between quantum states. Then we use it to classify quantum states, and such a classification is called a BSCC in Section 3.2. In the last section, we present the BSCC decomposition of quantum Markov chains. All these results come from [68].

\subsection{Reachability}

As we know in Chapter 1, the adjacency relation between states plays an irreplaceable role in the analysis of Markov chains. Actually, we can similarly define a quantum version.

Definition $13([68])$ : Let $\mathcal{G}=(\mathcal{H}, \mathcal{E})$ be a quantum Markov chain, and let $\rho$ and 
$\sigma$ be two quantum states.

- $\sigma$ is adjacent to $\rho$, written $\rho \rightarrow \sigma$ if $\operatorname{supp}(\sigma) \subseteq \operatorname{supp}(\mathcal{E}(\rho))$;

- $\sigma$ is reachable from $\rho$, if there is a path from $\rho$ to $\sigma$, i.e. there is a sequence $\rho \rightarrow \rho_{1} \rightarrow \cdots \rightarrow \rho_{m} \rightarrow \sigma$.

Therefore, starting from a state $\rho$, we can collect all reachable states together as a reachable space.

Definition $14([70])$ : Let $\mathcal{G}=(\mathcal{H}, \mathcal{E})$ be a quantum Markov chain. For any $\rho \in D(\mathcal{H})$, the reachable space of $\rho$ is defined to be

$$
\mathcal{R}_{\mathcal{G}}(\rho):=\bigvee_{i=0}^{\infty} \operatorname{supp}\left(\mathcal{E}^{i}(\rho)\right),
$$

where $\mathcal{E}^{i}$ stands for the composition of $i$ copies of $\mathcal{E}$, that is, $\mathcal{E}^{0}=\mathcal{I}$, the identity super-operator on $\mathcal{H}$, and $\mathcal{E}^{i}=\mathcal{E}^{i-1} \circ \mathcal{E}$ for $i \geq 1$.

Intuitively, as its name suggests, $\mathcal{R}_{\mathcal{G}}(\rho)$ consists of all states that can be reached from the initial state $\rho$ in the iterative evolution of the system modeled by $\mathcal{G}$. Surprisingly, this subspace can be computed in finite steps.

Lemma 7 ([70]): Let $\mathcal{G}=(\mathcal{H}, \mathcal{E})$ be a quantum Markov chain and $n=\operatorname{dim}(\mathcal{H})$. Then for any state $\rho \in D(\mathcal{H})$, we have

$$
\mathcal{R}_{\mathcal{G}}(\rho)=\bigvee_{i=0}^{n-1} \operatorname{supp}\left(\mathcal{E}^{i}(\rho)\right) .
$$

The above lemma indicates that all reachable states can be actually reached within $n$ steps if $\operatorname{dim}(\mathcal{H})=n$.

\subsection{Classification of Quantum States}

As in the classical case, the reachability can be used to group quantum states, and this will simplify the studies of quantum Markov chains in the latter discussion. 
A central concept in analysis of quantum Markov chains is the strongly connected component. Before giving its definition, let us first introduce an auxiliary notation. Let $\mathcal{X}$ be a subspace of a Hilbert space $\mathcal{H}$, and $\mathcal{E}$ be a super-operator on $\mathcal{H}$. Then the restriction of $\mathcal{E}$ on $\mathcal{X}$ is defined to be a super-operator $\left.\mathcal{E}\right|_{\mathcal{X}}$ with $\left.\mathcal{E}\right|_{\mathcal{X}}(\rho)=P_{\mathcal{X}} \mathcal{E}(\rho) P_{\mathcal{X}}$ for all $\rho \in D(\mathcal{X})$, where $P_{\mathcal{X}}$ is the projector onto $\mathcal{X}$; sometimes, we also use $\mathcal{E}_{\mathcal{X}}$ to replace $\left.\mathcal{E}\right|_{\mathcal{X}}$ when there is no confusion.

Definition $15([68])$ : Let $\mathcal{G}=(\mathcal{H}, \mathcal{E})$ be a quantum Markov chain. A subspace $\mathcal{X}$ of $\mathcal{H}$ is called strongly connected in $\mathcal{G}$ if for any $|\phi\rangle,|\psi\rangle \in \mathcal{X}$, we have $|\phi\rangle \in$ $\mathcal{R}_{\mathcal{G}_{\mathcal{X}}}(|\psi\rangle\langle\psi|)$ and $|\psi\rangle \in \mathcal{R}_{\mathcal{G}_{\mathcal{X}}}(|\phi\rangle\langle\phi|)$, where $\mathcal{G}_{\mathcal{X}}$ denotes the quantum Markov chain $\left(\mathcal{X},\left.\mathcal{E}\right|_{\mathcal{X}}\right)$; that is, $|\phi\rangle$ and $|\psi\rangle$ can be reached from each other.

Let $\mathrm{SC}(\mathcal{G})$ be the set of all strongly connected subspaces of $\mathcal{H}$ in $\mathcal{G}$. It is easy to see that the partial order $(\mathrm{SC}(\mathcal{G}), \subseteq)$ is inductive. Then Zorn's lemma asserts that it has maximal elements. Each maximal element of $(\mathrm{SC}(\mathcal{G}), \subseteq)$ is called a strongly connected component (SCC) of $\mathcal{G}$.

Definition $16([68])$ : Let $\mathcal{G}=(\mathcal{H}, \mathcal{E})$ be a quantum Markov chain. Then a subspace $\mathcal{X}$ of $\mathcal{H}$ is called a bottom strongly connected component (BSCC) of $\mathcal{G}$ if it is a SCC and invariant in $\mathcal{G}$. Here $\mathcal{X}$ is said to be invariant in $\mathcal{G}$ if $\mathcal{E}(\mathcal{X}) \subseteq \mathcal{X}$.

The following characterization of BSCCs establishes a connection between BSCCs and minimal stationary states.

Lemma 8 ([68]): A subspace $\mathcal{X}$ is a BSCC of quantum Markov chain $\mathcal{G}=(\mathcal{H}, \mathcal{E})$ if and only if there exists a minimal stationary state $\rho^{*}$ of $\mathcal{E}$ such that $\operatorname{supp}\left(\rho^{*}\right)=\mathcal{X}$.

Another important subspace, called a transient subspace, is necessary when we consider to decompose the state space $\mathcal{H}$ into BSCCs.

Definition $17([68])$ : Let $\mathcal{G}=(\mathcal{H}, \mathcal{E})$ be a quantum Markov chain. A subspace 
$\mathcal{X}$ of $\mathcal{H}$ is called a transient subspace if for any $\rho \in D(\mathcal{H})$,

$$
\lim _{n \rightarrow \infty} \operatorname{tr}\left(P_{\mathcal{X}} \mathcal{E}^{n}(\rho)\right)=0
$$

Intuitively, equation (3.1) means that the system will eventually go out of $\mathcal{X}$ no matter where it starts. Further, a transient subspace must be orthogonal to any BSCC, which is similar to the relation of transient state set and recurrent state set in classical Markov chains, i.e., a state must be precisely in one of them.

\subsection{BSCC Decomposition}

In the last section, we obtain two different subspaces according to reachability: BSCCs and transient subspaces. These two types of components motivate us to decompose the Hilbert state space as follows.

Theorem 3 (BSCC Decomposition, [68]): For any quantum Markov chain $\mathcal{G}=$ $(\mathcal{H}, \mathcal{E})$, we have:

$$
\mathcal{H}=\mathcal{B}_{0} \bigoplus \cdots \bigoplus \mathcal{B}_{n-1} \bigoplus \mathcal{T}_{\mathcal{E}}
$$

where $\bigoplus$ denotes direct sum, $\mathcal{B}_{i}{ }^{\prime}$ s are mutually orthogonal BSCCs of $\mathcal{G}$ and $\mathcal{T}_{\mathcal{E}}$ is the largest transient subspace in $\mathcal{G}$.

Unlike classical case, the BSCC decomposition is not unique, but we can conclude a relation between different decompositions.

Lemma 9 ([68]): Let $\mathcal{G}=(\mathcal{H}, \mathcal{E})$ be a quantum Markov chain. If we have two different BSCC decompositions

$$
\mathcal{H}=\mathcal{B}_{0} \bigoplus \cdots \bigoplus \mathcal{B}_{n-1} \bigoplus \mathcal{T}_{\mathcal{E}}=\mathcal{C}_{0} \bigoplus \cdots \bigoplus \mathcal{C}_{m-1} \bigoplus \mathcal{T}_{\mathcal{E}}
$$

where $\mathcal{B}_{i}$ 's and $\mathcal{C}_{i}$ 's are arranged, respectively, by the increasing order of the dimensions, then $m=n$, and $\operatorname{dim}\left(\mathcal{B}_{i}\right)=\operatorname{dim}\left(\mathcal{C}_{i}\right)$ for all $0 \leq i \leq n-1$.

The algorithm to implement the BSCC decomposition can also be found in [68]. 


\section{Chapter 4}

\section{Stationary Coherence Decomposition and Fixed Points}

In many fields, equilibria or stability are fundamental concepts that can be described regarding fixed points. In particular, fixed points of quantum Markov chains are important for stabilizing quantum subsystems and preparing quantum states [59, 61, 54]. The structure of them also gives us more effective ways to protect quantum information [9, 13]. Such a structure can be fully characterized by a decomposition, called stationary coherence decomposition.

In this chapter, our aim is to introduce the stationary coherence decomposition and show how this finds out all fixed points for a given quantum Markov chain; For more details, please see [5].

\subsection{Stationary Coherence Decomposition}

Stationary coherence, introduced in [5], is a key relation to classify BSCCs.

Definition 18 ([5]): Two mutually orthogonal BSCCs $\mathcal{B}_{1}$ and $\mathcal{B}_{2}$ in a quantum Markov chain $\mathcal{G}=(\mathcal{H}, \mathcal{E})$ have a stationary coherence if there is a BSCC $\mathcal{B}_{3} \subseteq$ $\mathcal{B}_{1} \oplus \mathcal{B}_{2}$ with $\mathcal{B}_{3} \neq \mathcal{B}_{1}, \mathcal{B}_{2}$

Stationary coherence is a genuinely quantum feature of quantum Markov chains, without a counterpart in classical Markov chains. We can group BSCCs by stationary coherences.

Theorem 4 (Stationary Coherence Decomposition, [5]): For any quantum Markov 
chain $\mathcal{G}=(\mathcal{H}, \mathcal{E})$, we have a unique orthogonal decomposition:

$$
\mathcal{H}=\bigoplus_{l} \mathcal{X}_{l} \bigoplus \mathcal{T}_{\mathcal{E}}
$$

where:

(1) $\mathcal{T}_{\mathcal{E}}$ is the largest transient subspace of $\mathcal{G}$.

(2) Each $\mathcal{X}_{l}$ is either a BSCC or can be further decomposed into mutually orthogonal BSCCs:

$$
\mathcal{X}_{l}=\mathcal{B}_{l, 0} \bigoplus \cdots \bigoplus \mathcal{B}_{l, n_{l}-1}
$$

such that

- all BSCCs $\mathcal{B}_{l, j}\left(0 \leq j<n_{l}\right)$ have the same dimension; and

- there are stationary coherences between any two of them.

(3) There are no stationary coherences between BSCCs in $\mathcal{X}_{i}$ and $\mathcal{X}_{j}$ if $i \neq j$.

In the last chapter, we have seen that unlike classical Markov chains, the BSCC decomposition is not unique in general for quantum Markov chains. By Theorem 4. we see that the stationary coherence between BSCCs is responsible for this nonuniqueness.

For practical applications, an algorithm to implement the two-level decomposition is crucial. Actually, we have already an algorithm to compute the BSCC decomposition in [68]. So, a key to computing the two-level composition is to identify the BSCCs having stationary coherences. This problem is solved by the following:

Lemma 10: Let $\mathcal{B}_{1}$ and $\mathcal{B}_{2}$ be two mutually orthogonal BSCCs of a quantum Markov chain $(\mathcal{H}, \mathcal{E})$ and $\mathcal{F}=\left\{X \in \mathcal{L}\left(\mathcal{B}_{1} \oplus \mathcal{B}_{2}\right) \mid \mathcal{E}(X)=X\right\}$. Then there is a stationary coherence between $\mathcal{B}_{1}$ and $\mathcal{B}_{2}$ if and only if $\operatorname{dim}(\mathcal{F})>2$. 
Proof. By Lemma $8, \mathcal{B} \subseteq \mathcal{H}$ is a BSCC if and only if there is a minimal stationary state $\rho$ such that $\operatorname{supp}(\rho)=\mathcal{B}$. If $\mathcal{B}_{1}$ and $\mathcal{B}_{2}$ are two mutually orthogonal BSCCs, let $\rho_{1} \in D\left(\mathcal{B}_{1}\right)$ and $\rho_{2} \in D\left(\mathcal{B}_{2}\right)$ be corresponding minimal stationary states. Then there is a stationary coherence if and only if there is at least one stationary state $\rho_{3}$ which is not a linear combination of $\rho_{1}$ and $\rho_{2}$. The lemma then follows from [66, Corollary 6.5].

We further observe that the stationary coherence is transitive in the sense that if there are stationary coherences between $\mathcal{B}_{1}$ and $\mathcal{B}_{2}$ and between $\mathcal{B}_{2}$ and $\mathcal{B}_{3}$, then there is a stationary coherence between $\mathcal{B}_{1}$ and $\mathcal{B}_{3}$ as well.

No algorithm for computing the stationary coherence decomposition has been given in the previous literature. We developed an algorithm for this purpose. It can be combined with the BSCC decomposition algorithm in [68] to compute the stationary coherence decomposition of quantum Markov chains. This combination is presented as Algorithm 1. The time complexity of Algorithm 1 is $O\left(n^{8}\right)$, where $\operatorname{dim}(\mathcal{H})=n$.

\subsection{Fixed Points}

To characterize $\operatorname{fix}(\mathcal{E})$, the main step is to study the Krause operators $\left\{E_{k}\right\}_{k}$ of $\mathcal{E}$ according to the stationary coherence.

Lemma 11 ([5]): Given a quantum Markov chain $\mathcal{G}=(\mathcal{H}, \mathcal{E})$, let $\mathcal{B}_{1}$ and $\mathcal{B}_{2}$ be two orthogonal BSCCs. Then there is a stationary coherence if and only if there is a unitary matrix $U$ such that $E_{k, 1}=U E_{k, 2} U^{\dagger}$ for all $k$, where $\left\{E_{k, i}\right\}_{k}$ are the restriction of Kraus operators $\left\{E_{k}\right\}_{k}$ of $\mathcal{E}$ onto $\mathcal{B}_{i}$ for $i=1$, 2, i.e. $E_{k, i}=P_{\mathcal{B}_{i}} E_{k} P_{\mathcal{B}_{i}}$. Furthermore, $\mathcal{B}_{1} \simeq \mathcal{B}_{2}$.

The above lemma tells us that in some basis, all mutually orthogonal BSCCs with stationary coherences are identical. Through this observation, we can get a 


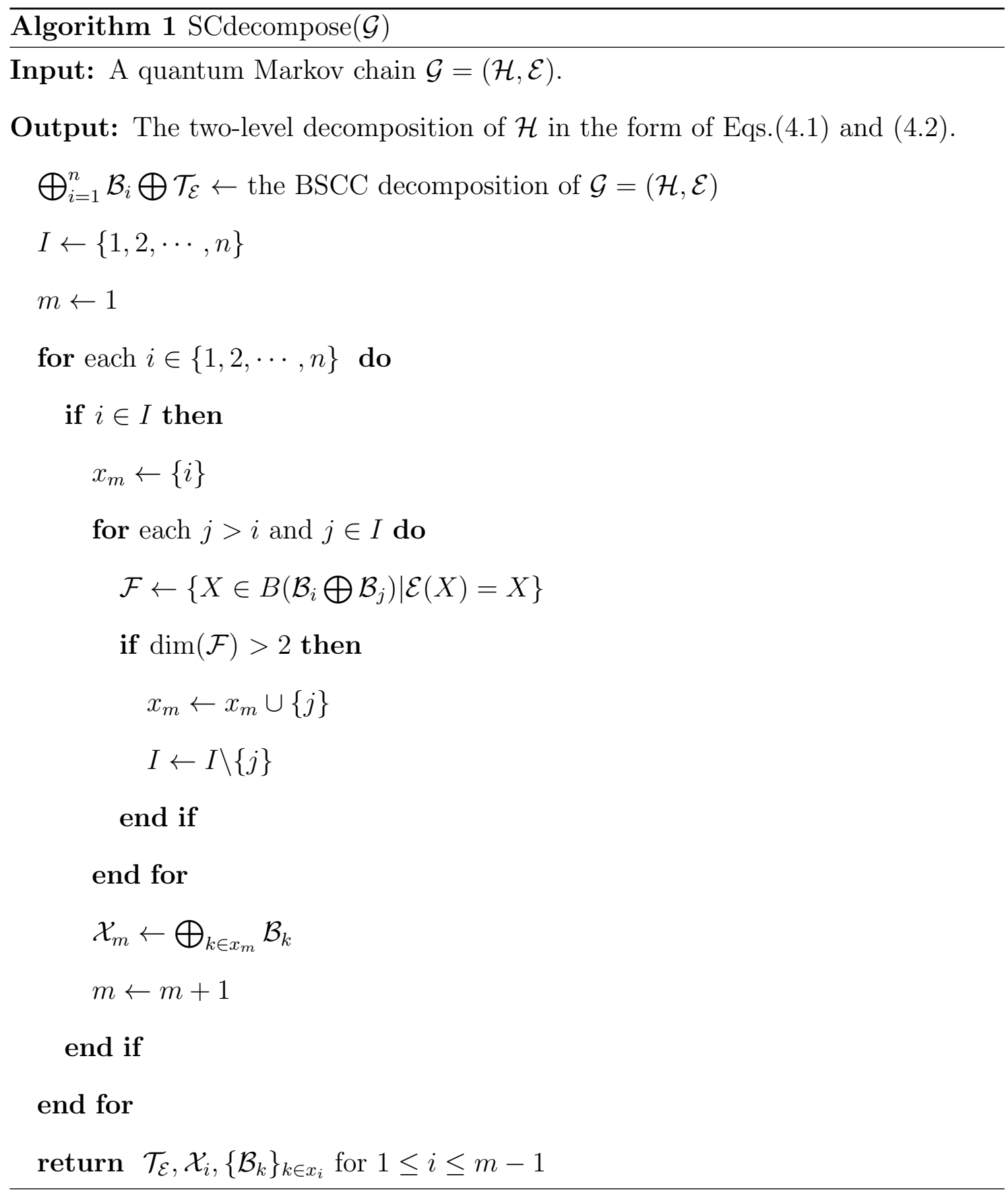


structure of $f i x(\mathcal{E})$ as follows.

Theorem 5 (fixed-point decomposition, [5]): Let $\mathcal{E}$ be a super-operator on $\mathcal{H}$. Then there is a unique orthogonal decomposition of $\mathcal{H}$

$$
\mathcal{H}=\bigoplus_{l=1}^{L} \mathcal{X}_{l} \oplus \mathcal{T}_{\mathcal{E}}
$$

where:

(1) $\mathcal{T}_{\mathcal{E}}$ is the largest transient subspace;

(2) each $\mathcal{X}_{l}$ is either a BSCC or can be further decomposed into mutually orthogonal BSCCs with stationary coherences between any two of them:

$$
\mathcal{X}_{l}=\bigoplus_{p=1}^{m_{l}} \mathcal{B}_{l, p} \simeq \mathbb{C}^{m_{l}} \otimes \mathcal{B}_{l}, \quad \mathcal{B}_{l} \simeq \mathcal{B}_{l, p} \forall p
$$

so that the Kraus operators $\left\{E_{k}\right\}$ of $\mathcal{E}$ have a block form

$$
E_{k} \simeq\left[\begin{array}{ccc|c}
I_{1} \otimes E_{k, 1} & & & \\
& \ddots & & T_{k} \\
& & I_{L} \otimes E_{k, L} & \\
\hline & & & K_{k}
\end{array}\right]
$$

in the corresponding basis, for some operators $E_{k, l} \in \mathcal{L}\left(\mathcal{B}_{l}\right), K_{k} \in \mathcal{L}(\mathcal{K})$, and $T_{k} \in \mathcal{L}\left(\mathcal{K}, \mathcal{K}^{\perp}\right)$. Here $I_{l}$ is the identity operator on $\mathbb{C}^{m_{l}}$ and $\mathcal{E}_{l}(\cdot)=$ $\sum_{k} E_{k, l} \cdot E_{k, l}^{\dagger}$ has only one stationary state in $D\left(\mathcal{B}_{l}\right)$ and the state is full-rank on $\mathcal{B}_{l}$. Furthermore,

$$
f i x(\mathcal{E}) \simeq \bigoplus_{l}\left[\mathcal{L}\left(\mathbb{C}^{m_{l}}\right) \otimes \rho_{l}\right] \oplus 0_{\mathcal{K}}
$$

where $\rho_{l}$ is the unique stationary state of $\mathcal{E}_{l}$, and $0_{\mathcal{K}}$ is the zero operator on $\mathcal{K}$.

(3) there is no stationary coherence between any BSCCs $\mathcal{B}_{l, p}$ and $\mathcal{B}_{l^{\prime}, p^{\prime}}$ whenever $l \neq l^{\prime}$. 
This decomposition is unique and called the fixed-point decomposition of $\mathcal{H}$ and can be computed by applying the structure of $C^{*}$-algebra generated by the Kraus operators of $\mathcal{E}$; see [33, 25, 63] for details. 


\section{Chapter 5}

\section{Periodic Decomposition and Limiting States}

A two-level decomposition of quantum Markov chains was developed in the previous chapters. In this chapter, we propose another decomposition technique, namely periodic decomposition, which can be combined with the two-level decomposition to form a three-level decomposition further. Such a three-level decomposition provides us with a handy tool for a finer algorithmic analysis of quantum Markov chains. Meanwhile, we give several characterizations of limiting states of quantum Markov chains concerning aperiodicity, irreducibility, and eigenvalues. These results are based on our recent paper [25].

\subsection{Irreducibility and Periodicity}

In this section, we first extend the notion of irreducibility for quantum Markov chains, which turns out to be equivalent to the irreducibility defined in the previous literature. Recall from classical probability theory that an irreducible Markov chain starting from a state can reach any other state in a finite number of steps. With the help of the reachable space introduced in Definition 14, we have:

Definition 19: A quantum Markov chain $\mathcal{G}=(\mathcal{H}, \mathcal{E})$ is called irreducible if for any $\rho \in D(\mathcal{H}), \mathcal{R}_{\mathcal{G}}(\rho)=\mathcal{H}$.

From Lemma 7, it can be easily shown that the above definition indeed coincides with the irreducibility given in [19] for quantum stochastic processes and [66, Theorem 6.2] for quantum channels. However, our definition presents a more natural extension of irreducibility for classical Markov chains. 
To illustrate irreducibility, let us see two simple examples.

Example 1: Consider a natural way to encode the classical NOT gate $X: 0 \rightarrow$ $1 ; 1 \rightarrow 0$ into a quantum super-operator. Let $\mathcal{H}=\operatorname{lin} . \operatorname{span}\{|0\rangle,|1\rangle\}$. The superoperator $\mathcal{E}: D(\mathcal{H}) \rightarrow D(\mathcal{H})$ is defined by

$$
\mathcal{E}(\rho)=|1\rangle\langle 0|\rho| 0\rangle\langle 1|+| 0\rangle\langle 1|\rho| 1\rangle\langle 0|
$$

for any $\rho \in D(\mathcal{H})$. It is easy to check that the quantum Markov chain $(\mathcal{H}, \mathcal{E})$ is irreducible.

Example 2 (Amplitude-damping channel): Consider the 2-dimensional amplitudedamping channel modeling the physical processes such as spontaneous emission. Let $\mathcal{H}=\operatorname{lin} . \operatorname{span}\{|0\rangle,|1\rangle\}$, and

$$
\mathcal{E}(\rho)=E_{0} \rho E_{0}^{\dagger}+E_{1} \rho E_{1}^{\dagger}
$$

where $E_{0}=|0\rangle\langle 0|+\sqrt{1-p}| 1\rangle\langle 1|$ and $E_{1}=\sqrt{p}|0\rangle\langle 1|$ with $p>0$. Then the quantum Markov chain $\mathcal{G}=(\mathcal{H}, \mathcal{E})$ is reducible since, say, $\mathcal{R}_{\mathcal{G}}(|0\rangle\langle 0|)=\operatorname{lin} . \operatorname{span}\{|0\rangle\}$.

Let us now consider how to check whether a quantum Markov chain $\mathcal{G}=(\mathcal{H}, \mathcal{E})$ is irreducible. Note from the BSCC decomposition that $\mathcal{G}$ is irreducible if and only if the state Hilbert space $\mathcal{H}$ itself is a BSCC of $\mathcal{G}$. Moreover, we have the following:

Lemma 12: A quantum Markov chain $\mathcal{G}=(\mathcal{H}, \mathcal{E})$ has a unique BSCC $\mathcal{B}$ if and only if it has a unique stationary state $\rho^{*}$. Furthermore, $\operatorname{supp}\left(\rho^{*}\right)=\mathcal{B}$.

Proof. We see from Definition 17 that for any stationary state $\rho, \operatorname{supp}(\rho) \subseteq \mathcal{B}$. Then the result immediately follows from Lemma 8 and Theorem 3 .

Therefore, the uniqueness of BSCCs in $\mathcal{G}$ can be used to check irreducibility of $\mathcal{G}$.

Theorem $6([19$, Theorem 13]): A quantum Markov chain $\mathcal{G}=(\mathcal{H}, \mathcal{E})$ is irreducible if and only if it has a unique stationary state $\rho^{*}$ with $\operatorname{supp}\left(\rho^{*}\right)=\mathcal{H}$. 
Several different versions of this theorem and its special cases are known in [66] and [21]. However, the above version can be more conveniently used in checking irreducibility of quantum Markov chains. Indeed, it shows that checking whether $\mathcal{G}=(\mathcal{H}, \mathcal{E})$ is irreducible can be done by Algorithm 1 in 68 to check whether its state space $\mathcal{H}$ is a BSCC. The time complexity is $O\left(n^{6}\right)$, where $\operatorname{dim}(\mathcal{H})=n$.

Next, we consider the periodicity of quantum Markov chains.

Definition 20: Let $\mathcal{G}=(\mathcal{H}, \mathcal{E})$ be a quantum Markov chain.

(1) A state $\rho \in D(\mathcal{H})$ is called aperiodic if

$$
\operatorname{gcd}\left\{m \geq 1: \operatorname{supp}(\rho) \subseteq \operatorname{supp}\left(\mathcal{E}^{m}(\rho)\right)\right\}=1
$$

Here, recall that ged stands for the greatest common divisor; in particular, we assume that $\operatorname{gcd}(\emptyset)=0$.

(2) A subspace $\mathcal{X}$ of $\mathcal{H}$ is aperiodic if each density operator $\rho$ with $\operatorname{supp}(\rho) \subseteq \mathcal{X}$ is aperiodic.

(3) If there exists an integer $d \geq 1$ such that the whole state space $\mathcal{H}$ is aperiodic in quantum Markov chain $\mathcal{G}^{d}=\left(\mathcal{H}, \mathcal{E}^{d}\right)$, then the minimum of such integers $d$, denoted $d(\mathcal{G})$, is called the period of $\mathcal{G}$.

(4) When $d(\mathcal{G})=1, \mathcal{G}$ is said to be aperiodic; otherwise, it is periodic.

For the special case of irreducible quantum Markov chains, periodicity was defined in [21, 11] based on the notion of $\mathcal{E}$-cyclic resolution:

Definition $21([11])$ : For a quantum Markov chain $\mathcal{G}=(\mathcal{H}, \mathcal{E})$, let $\left(P_{0}, \cdots, P_{d-1}\right)$ be a resolution of identity, i.e. a family of orthogonal projectors such that $\sum_{k=0}^{d-1} P_{k}=$ I. Then $\left(P_{0}, \cdots, P_{d-1}\right)$ is said to be $\mathcal{E}$-cyclic if $\mathcal{E}^{\dagger}\left(P_{k}\right)=P_{k \boxminus 1}$ for $k=0, \cdots, d-1$, where $\boxminus$ denotes subtraction modulo $d$ and $\mathcal{E}^{\dagger}$ is the adjoint map of $\mathcal{E}$; that is, the linear map such that $\operatorname{tr}(M \mathcal{E}(A))=\operatorname{tr}\left(\mathcal{E}^{\dagger}(M) A\right)$ for all $M$ and $A$ in $\mathcal{L}(\mathcal{H})$. 
The next lemma shows that the period defined in [11] and that in Definition 20 are the same for irreducible quantum Markov chains. Actually, the former can be better understood in the Heisenberg picture, and the latter in the Schrödinger picture.

Lemma 13: For an irreducible quantum Markov chain $\mathcal{G}=(\mathcal{H}, \mathcal{E})$, the period of $\mathcal{G}$ is equal to the maximum integer $c$ for which there exists a $\mathcal{E}$-cyclic resolution $\left(P_{0}, \cdots, P_{c-1}\right)$ of identity.

Proof. By [66, Theorem 6.6], the maximum $c$ for which there exists a $\mathcal{E}$-cyclic resolution $\left(P_{0}, \cdots, P_{c-1}\right)$ of identity is the number of the eigenvalues of $\mathcal{E}$ with magnitude one. Then the result follows from Lemma 17.

The notion of periodicity is further illustrated by the following example.

Example 3: Let $\mathcal{G}=(\mathcal{H}, \mathcal{E})$ with $\mathcal{H}=\operatorname{lin} . \operatorname{span}\{|0\rangle,|1\rangle,|2\rangle\}$ and for any $\rho \in$ $D(\mathcal{H})$

$$
\mathcal{E}(\rho)=|1+2\rangle\langle 0|\rho| 0\rangle\langle 1+2|+| 0+2\rangle\langle 1|\rho| 1\rangle\langle 0+2|+| 1+0\rangle\langle 2|\rho| 2\rangle\langle 1+0|
$$

where $|i+j\rangle=(|i\rangle+|j\rangle) / \sqrt{2}$ for $i, j \in\{0,1,2\}$. Then it is easy to see that $\mathcal{G}$ is irreducible and aperiodic, and has the unique stationary state

$$
\frac{1}{3}(|1+2\rangle\langle 1+2|+| 0+2\rangle\langle 0+2|+| 1+0\rangle\langle 1+0|) .
$$

The following lemma presents a useful characterization of the reachable space starting from a state within an aperiodic subspace. It can be seen as a strengthened version of Lemma 7 in the special case of aperiodic quantum Markov chains.

Lemma 14: Let $\mathcal{G}=(\mathcal{H}, \mathcal{E})$ be a quantum Markov chain and $\mathcal{X}$ be a subspace of $\mathcal{H}$. Then the following statements are equivalent:

(1) $\mathcal{X}$ is an aperiodic subspace of $\mathcal{H}$; 
(2) For any $\rho \in D(\mathcal{H})$ with $\operatorname{supp}(\rho) \subseteq \mathcal{X}$, there exists an integer $M(\rho)>0$ such that $\operatorname{supp}\left(\mathcal{E}^{m}(\rho)\right)=\mathcal{R}_{\mathcal{G}}(\rho)$ for all $m \geq M(\rho)$.

Proof. $(2) \Rightarrow(1)$ is obvious. So, we only need to show that $(1) \Rightarrow(2)$. Fix an arbitrary $\rho$ with $\operatorname{supp}(\rho) \subseteq \mathcal{X}$. For each $i \geq 0$, let $\mathcal{X}_{i}=\operatorname{supp}\left(\mathcal{E}^{i}(\rho)\right)$. In particular, $\mathcal{X}_{0}=\operatorname{supp}(\rho)$. Let $T_{\rho}=\left\{i \geq 1: \mathcal{X}_{i} \supseteq \mathcal{X}_{0}\right\}$. Then from Lemma 4 , we have: for any $i, j \geq 0$

$$
\begin{aligned}
& \mathcal{X}_{i+j}=\mathcal{E}^{i}\left(\mathcal{X}_{j}\right) \text {; and } \\
& \text { if } i, j \in T_{\rho} \text {, then } i+j \in T_{\rho} .
\end{aligned}
$$

By the assumption that $\mathcal{X}$ is aperiodic, we have $\operatorname{gcd}\left(T_{\rho}\right)=1$. Then from [40], there is a finite subset $\left\{m_{k}\right\}_{k \in K}$ of $T_{\rho}, \operatorname{gcd}\left\{m_{k}\right\}_{k \in K}=1$, and an integer $M^{\prime}(\rho)>0$ such that for any $i \geq M^{\prime}(\rho)$, there exist positive integers $\left\{a_{k}\right\}_{k \in K}$ such that $i=\sum_{k \in K} a_{k} m_{k}$. Thus $i \in T_{\rho}$ from Eq. (5.2).

Now let $M(\rho)=M^{\prime}(\rho)+n-1$ where $n=\operatorname{dim}(\mathcal{H})$, and take any $m \geq M(\rho)$. For all $0 \leq i \leq n-1$, we have shown that $m-i \in T_{\rho}$; that is, $\mathcal{X}_{m-i} \supseteq \mathcal{X}_{0}$. Thus $\mathcal{X}_{m} \supseteq \mathcal{X}_{i}$ from Eq. (5.1), and $\mathcal{X}_{m} \supseteq \mathcal{R}_{\mathcal{G}}(\rho)$ from Lemma 7. Therefore, $\mathcal{X}_{m}=\mathcal{R}_{\mathcal{G}}(\rho)$, as the reverse inclusion trivially holds.

Combining the above lemma with Definition 19 , we have:

Corollary 1: Let $\mathcal{G}=(\mathcal{H}, \mathcal{E})$ be an irreducible and aperiodic quantum Markov chain. Then for any $\rho \in D(\mathcal{H})$, there exists an integer $M(\rho)>0$ such that $\operatorname{supp}\left(\mathcal{E}^{m}(\rho)\right)=\mathcal{H}$ for all $m \geq M(\rho)$.

The above corollary shows that starting from any state $\rho$, an irreducible and aperiodic quantum Markov chain can reach the whole state space after a finite number of steps. Then it is interesting to see when the whole space can be reached for the first time.

Definition 22: Let $\mathcal{G}=(\mathcal{H}, \mathcal{E})$ be an irreducible and aperiodic quantum Markov 
chain. For each $\rho \in D(\mathcal{H})$, the saturation time of $\rho$ is defined to be

$$
s(\rho)=\inf \left\{n \geq 1 \mid \operatorname{supp}\left(\mathcal{E}^{n}(\rho)\right)=\mathcal{H}\right\} .
$$

It is clear from Corollary 1 that the infimum in the defining equation of $s(\rho)$ can always be attained. Furthermore, we can show that for an irreducible and aperiodic quantum Markov chain, the saturation time for any initial state has a universal upper bound.

Lemma 15: Let $\mathcal{G}=(\mathcal{H}, \mathcal{E})$ be a quantum Markov chain and $\mathcal{X}$ be an invariant subspace of $\mathcal{H}$. Then the following statements are equivalent:

(1) $\mathcal{G}_{\mathcal{X}}=\left(\mathcal{X},\left.\mathcal{E}\right|_{\mathcal{X}}\right)$ is irreducible and aperiodic;

(2) There exists an integer $M>0$ such that for all $\rho \in D(\mathcal{X}), \operatorname{supp}\left(\mathcal{E}^{m}(\rho)\right)=\mathcal{X}$ for all $m \geq M$.

Proof. $(2) \Rightarrow(1)$ is obvious. So, we only need to show that $(1) \Rightarrow(2)$. Let $s_{\mathcal{X}}(\rho)$ be the saturation time of $\rho$ in $\mathcal{G}_{\mathcal{X}}$. Then for any $\rho \in D(\mathcal{X})$, let

$$
B(\rho)=\left\{\sigma \in D(\mathcal{X}) \mid\|\rho-\sigma\|_{1}<\bar{\lambda}_{\min }\left(\mathcal{E}^{s \mathcal{X}(\rho)}(\rho)\right)\right\}
$$

where $\|\cdot\|_{1}$ is the trace norm and $\bar{\lambda}_{\min }(\rho)$ is the minimum non-zero eigenvalue of $\rho$. Obviously, $B(\rho)$ is an open set. Then $\{B(\rho)\}_{\rho \in D(\mathcal{X})}$ is an open cover of $D(\mathcal{X})$. As $D(\mathcal{X})$ is compact, we can find a finite number of density operators $\left\{\rho_{i}\right\}_{i \in J}$ such that

$$
D(X)=\bigcup_{i \in J} B\left(\rho_{i}\right)
$$

In the following, we show for any $\rho \in D(\mathcal{X})$ and $\sigma \in B(\rho), \operatorname{supp}\left(\mathcal{E}^{m}(\sigma)\right)=\mathcal{X}$ for all $m \geq s(\rho)$. Then the theorem holds by taking $M=\max _{i \in J} s_{\mathcal{X}}\left(\rho_{i}\right)$. Let $\mathcal{Y}=\operatorname{supp}\left(\mathcal{E}^{s_{\mathcal{X}}(\rho)}(\sigma)\right)$, and $P_{\mathcal{Y}}$ be the projector onto $\mathcal{Y}$. As $\mathcal{X}$ is invariant, $\mathcal{Y} \subseteq \mathcal{X}$. 
Let $P_{\overline{\mathcal{Y}}}=I_{\mathcal{X}}-P_{\mathcal{Y}}$, where $I_{\mathcal{X}}$ is the identity operator on $\mathcal{X}$. Then

$$
\begin{aligned}
\operatorname{tr}\left(P_{\overline{\mathcal{Y}}} \mathcal{E}^{s_{\mathcal{X}}(\rho)}(\rho)\right) & =\left\|P_{\overline{\mathcal{Y}}} \mathcal{E}^{s_{\mathcal{X}}(\rho)}(\rho) P_{\overline{\mathcal{Y}}}\right\|_{1} \\
& =\left\|P_{\overline{\mathcal{Y}}}\left(\mathcal{E}^{s_{\mathcal{X}}(\rho)}(\rho)-\mathcal{E}^{s_{\mathcal{X}}(\rho)}(\sigma)\right) P_{\overline{\mathcal{Y}}}\right\|_{1} \\
& \leq\left\|\mathcal{E}^{s_{\mathcal{X}}(\rho)}(\rho)-\mathcal{E}^{s_{\mathcal{X}}(\rho)}(\sigma)\right\|_{1} \\
& \leq\|\rho-\sigma\|_{1} \\
& <\bar{\lambda}_{\text {min }}\left(\mathcal{E}^{s_{\mathcal{X}}(\rho)}(\rho)\right)
\end{aligned}
$$

The first two inequalities follow from Lemma 5. By Lemma 6, this is only possible when $\mathcal{Y}=\mathcal{X}$, since $\mathcal{X}$ is invariant. In other words, $\operatorname{supp}\left(\mathcal{E}^{s \mathcal{X}(\rho)}(\sigma)\right)=\mathcal{X}$. Thus $\operatorname{supp}\left(\mathcal{E}^{s_{\mathcal{X}}(\rho)-1}(\sigma)\right) \subseteq \operatorname{supp}\left(\mathcal{E}^{s_{\mathcal{X}}(\rho)}(\sigma)\right)$, and $\operatorname{supp}\left(\mathcal{E}^{s_{\mathcal{X}}(\rho)}(\sigma)\right) \subseteq \operatorname{supp}\left(\mathcal{E}^{s_{\mathcal{X}}(\rho)+1}(\sigma)\right)$ from Lemma 4. So

$$
\operatorname{supp}\left(\mathcal{E}^{s \mathcal{X}(\rho)+1}(\sigma)\right)=\mathcal{X}
$$

By induction, we can show that $\operatorname{supp}\left(\mathcal{E}^{m}(\sigma)\right)=\mathcal{X}$ for all $m \geq s_{\mathcal{X}}(\rho)$.

It is worth noting that the integer $M$ in the above theorem does not depend on state $\rho$. This makes it much stronger than Lemma 14. Considering the whole state space, we have:

Corollary 2: Let $\mathcal{G}=(\mathcal{H}, \mathcal{E})$ be a quantum Markov chain. Then the following statements are equivalent:

(1) $\mathcal{G}$ is irreducible and aperiodic;

(2) There exists an integer $M>0$ such that for all $\rho \in D(\mathcal{H}), \operatorname{supp}\left(\mathcal{E}^{m}(\rho)\right)=\mathcal{H}$ for all $m \geq M$.

Note that a (classical) Markov chains described by a stochastic $k$-by- $k$ matrix $P$ is irreducible and aperiodic if and only if there exists an integer $m$ such that $\left(P^{m}\right)_{i, j}>0$ for all $i$ and $j$. Then by (classical) Perron-Frobenius theory, we have Wielandt's inequality [65]: the minimum $m \leq k^{2}-2 k+2$. A quantum Wielandt's 
inequality was recently proved in [53]. As its direct application, we see that the minimal $M$ in Corollary 2 satisfies $M \leq n^{4}$ where $n=\operatorname{dim}(\mathcal{H})$. Then a limit theorem of quantum Markov chain can be directly obtained by combining Corollary 2 and [66. Theorem 6.7].

Theorem 7 (Limit Theorem): Let $\mathcal{G}=(\mathcal{H}, \mathcal{E})$ be a quantum Markov chain. Then the following statements are equivalent:

(1) $\mathcal{G}$ has a limiting state $\rho^{*}$ with $\operatorname{supp}\left(\rho^{*}\right)=\mathcal{H}$ in the sense that

$$
\lim _{n \rightarrow \infty} \mathcal{E}^{n}(\rho)=\rho^{*}, \forall \rho \in \mathcal{D}(\mathcal{H})
$$

(2) $\mathcal{G}$ is irreducible and aperiodic;

(3) 1 is the only eigenvalue of $\mathcal{E}$ with magnitude one and the corresponding eigenvector $\rho^{*}$ is positive definite.

Proof. Direct from [66, Theorem 6.7], by noting that irreducibility plus aperiodicity are equivalent to primitivity with Corollary 2 .

Generally, aperiodicity can be determined by the eigenvalues of $\mathcal{E}$ without the assumption of irreducibility.

Lemma 16: Let $\mathcal{G}=(\mathcal{H}, \mathcal{E})$ be a quantum Markov chain with a trivial transient subspace; that is, $\mathcal{T}_{\mathcal{E}}=\{0\}$ in the decomposition in Eq. 3.2 . If $\mathcal{E}$ has only 1 as its eigenvalue with magnitude one, then $\mathcal{G}$ must be aperiodic.

Proof. As 1 is the only eigenvalue with magnitude one, $\mathcal{E}_{\phi}=\lim _{n \rightarrow \infty} \mathcal{E}^{n}$. Then for any $|\psi\rangle \in \mathcal{H}$,

$$
\lim _{n \rightarrow \infty} \mathcal{E}^{n}(|\psi\rangle\langle\psi|)=\rho^{*}
$$

for some stationary state $\rho^{*}$.

By the proof of Lemma 15, there exists an integer $N>0$ such that for all $n>N$,

$$
\operatorname{supp}\left(\mathcal{E}^{n}(|\psi\rangle\langle\psi|)\right)=\operatorname{supp}\left(\rho^{*}\right)
$$


Then with $\mathcal{T}_{\mathcal{E}}=\{0\}$ and Lemma 8 , there is a stationary state $\sigma^{*}$ such that $\operatorname{tr}\left(\sigma^{*} \rho^{*}\right)=$ 0 and $\operatorname{supp}\left(\rho^{*}+\sigma^{*}\right)=\mathcal{H}$. Then as $\operatorname{supp}\left(\rho^{*}\right), \operatorname{supp}\left(\sigma^{*}\right)$ is invariant under $\mathcal{E}_{\phi}$ and $\mathcal{E}_{\phi}=$ $\lim _{n \rightarrow \infty} \mathcal{E}^{n}$ is CPTP, it is easy to see that $|\psi\rangle \in \operatorname{supp}\left(\rho^{*}\right)$. Therefore, by Definition 20, $|\psi\rangle\langle\psi|$ is aperiodic. Consequently, $(\mathcal{H}, \mathcal{E})$ is aperiodic from the arbitrariness of $|\psi\rangle$.

The next lemma shows that the period of an irreducible quantum Markov chain is exactly the number of eigenvalues with magnitude one.

Lemma 17: For an irreducible quantum Markov chain $\mathcal{G}=(\mathcal{H}, \mathcal{E})$, the period of $\mathcal{G}$ equals the number of eigenvalues of $\mathcal{E}$ with magnitude one.

Proof. Let $m$ be the number of eigenvalues of $\mathcal{E}$ with magnitude one and $d$ the period of $\mathcal{G}$. By [66, Theorem 6.6], 1 is the only element in $\operatorname{spec}\left(\mathcal{E}^{m}\right)$ with magnitude one. As $\mathcal{G}$ is irreducible, $\mathcal{G}^{m}=\left(\mathcal{H}, \mathcal{E}^{m}\right)$ has only a trivial transient subspace by Theorem 6. Then from Lemma 16, $\mathcal{G}^{m}=\left(\mathcal{H}, \mathcal{E}^{m}\right)$ is aperiodic, and hence $d \leq m$.

We now turn to prove that $d \geq m$. The case when $m=1$ is trivial. Suppose $m \geq$ 2. By [66, Theorem 6.6], there exists a $\mathcal{E}$-cyclic resolution of identity $\left(P_{0}, \cdots, P_{m-1}\right)$. As $\mathcal{G}^{d}=\left(\mathcal{H}, \mathcal{E}^{d}\right)$ is aperiodic, there exists an integer $N^{\prime}>0$ such that $\operatorname{supp}\left(P_{k}\right) \subseteq$ $\operatorname{supp}\left(\mathcal{E}^{d n}\left(P_{k}\right)\right)$ for all $n \geq N^{\prime}$. Thus for $n \geq N^{\prime}$

$$
0<\operatorname{tr}\left(P_{k} \mathcal{E}^{d n}\left(P_{k}\right)\right)=\operatorname{tr}\left(\mathcal{E}^{\dagger^{d n}}\left(P_{k}\right) P_{k}\right)=\operatorname{tr}\left(P_{k \boxminus d n} P_{k}\right)
$$

where $\boxminus$ denotes subtraction modulo $m$. Therefore, $m$ must be a factor of $d$ and $m \leq d$

Lemma 17 indicates that every irreducible quantum Markov chain has a period and also offers an efficient algorithm for computing the period by counting the number of eigenvalues of the super-operator. 


\subsection{Periodic Decomposition}

Theorem 8 (Periodic Decomposition): The state Hilbert space $\mathcal{H}$ of an irreducible quantum Markov chain $\mathcal{G}=(\mathcal{H}, \mathcal{E})$ with period $d$ can be decomposed into the direct sum of some orthogonal subspaces:

$$
\mathcal{H}=\mathcal{B}_{0} \bigoplus \cdots \bigoplus \mathcal{B}_{d-1}
$$

with the following properties:

(1) $\mathcal{E}\left(\mathcal{B}_{i \boxminus 1}\right)=\mathcal{B}_{i}$, where $\boxminus$ denotes subtraction modulo $d$;

(2) $\left(\mathcal{B}_{i},\left.\mathcal{E}^{d}\right|_{\mathcal{B}_{i}}\right)$ is irreducible and aperiodic; and

(3) $\mathcal{B}_{i}{ }^{\prime}$ s are mutually orthogonal subspaces of $\mathcal{H}$ and invariant under $\mathcal{E}^{d}$.

Proof. Immediate from the proof of Lemma 17.

Now we turn to integrate it with the two-level decomposition to form a finer decomposition of a general quantum Markov chain that might be reducible. For any quantum Markov chain $\mathcal{G}=(\mathcal{H}, \mathcal{E})$, we first use Theorem 3 to decompose $\mathcal{H}$ into

$$
\mathcal{H}=\mathcal{X}_{0} \bigoplus \cdots \bigoplus \mathcal{X}_{n-1} \bigoplus \mathcal{T}_{\mathcal{E}}
$$

where each $\mathcal{X}_{l}=\mathcal{B}_{l, 0} \bigoplus \cdots \bigoplus \mathcal{B}_{l, n_{l}-1}$. It was proved in [68] that although the BSCC decomposition of $\mathcal{H}$ is not unique, the number $n_{l}$ of BSCCs for different decompositions of $\mathcal{X}_{l}$ is the same. Furthermore, for each $\mathcal{B}_{i, l}$, we can employ Theorem 8 to decompose it into $d_{i, l}$ aperiodic subspaces, where $d_{i, l}$ is the period of $\mathcal{B}_{i, l}$. Then the only question that remains to answer is: is the sum $\sum_{i} d_{i, l}$ of the periods of BSCCs the same for different decompositions of $\mathcal{X}_{l}$ ? The following is a key lemma to give a positive answer to this question:

Lemma 18: Let $\mathcal{G}=(\mathcal{H}, \mathcal{E})$ be a quantum Markov chain with the stationary coherence decomposition:

$$
\mathcal{H}=\bigoplus_{l} \mathcal{X}_{l} \bigoplus \mathcal{T}_{\mathcal{E}}
$$


Then for any $l$ and any BSCCs $X$ and $Y$ contained in $\mathcal{X}_{l}$, we have $d(X)=d(Y)$, where $d(\cdot)$ denotes the period of $\mathcal{E}$ when restricting to the corresponding subspace.

Proof. Let $\mathcal{R}=\bigoplus_{l} \mathcal{X}_{l}$ be the subspace of $\mathcal{H}$ spanned by all BSCCs. For any subspace $Z$ of $\mathcal{H}$, let $P_{Z}$ be the projector onto $Z$ and $\mathcal{P}_{Z}$ be super-operator with $\mathcal{P}_{Z}(\cdot)=P_{Z} \cdot P_{Z}$. Then $\left.\mathcal{E}\right|_{Z}=\mathcal{P}_{Z} \circ \mathcal{E} \circ \mathcal{P}_{Z}$. By [5, Corollary 23], we can find a unitary $U$ such that

(1) $P_{Y}=U P_{X} U^{\dagger}$ (thus $\mathcal{P}_{Y}=\mathcal{U} \circ \mathcal{P}_{X} \circ \mathcal{U}^{\dagger}$ where $\mathcal{U}(\cdot)=U \cdot U^{\dagger}$ ); and

(2) for any linear operator $A$ with $A=\mathcal{P}_{\mathcal{R}}(A), \mathcal{P}_{\mathcal{R}} \circ \mathcal{E}^{\dagger} \circ \mathcal{U}(A)=\mathcal{U} \circ \mathcal{P}_{\mathcal{R}} \circ \mathcal{E}^{\dagger}(A)$.

For any orthogonal projectors $P_{0}, \cdots, P_{d-1}$ such that $\sum_{i=0}^{d-1} P_{i}=P_{X}$ and $\mathcal{E}_{X}^{\dagger}\left(P_{i}\right)=$ $\mathcal{P}_{X} \circ \mathcal{E}^{\dagger}\left(P_{i}\right)=P_{i \boxminus 1}$, where $\boxminus$ denotes subtraction modulo $d$, let $P_{0}^{\prime}, \cdots, P_{d-1}^{\prime}$ be orthogonal projectors with $P_{i}^{\prime}=\mathcal{U}\left(P_{i}\right)$. Then for any $i$,

$$
\begin{aligned}
\left(\left.\mathcal{E}\right|_{Y}\right)^{\dagger}\left(P_{i}^{\prime}\right) & =\mathcal{P}_{Y} \circ \mathcal{E}^{\dagger} \circ \mathcal{P}_{Y} \circ \mathcal{U}\left(P_{i}\right)=\mathcal{P}_{Y} \circ \mathcal{P}_{\mathcal{R}} \circ \mathcal{E}^{\dagger} \circ \mathcal{U}\left(P_{i}\right) \\
& =\mathcal{P}_{Y} \circ \mathcal{U} \circ \mathcal{P}_{\mathcal{R}} \circ \mathcal{E}^{\dagger}\left(P_{i}\right)=\mathcal{U} \circ \mathcal{P}_{X} \circ \mathcal{P}_{\mathcal{R}} \circ \mathcal{E}^{\dagger}\left(P_{i}\right) \\
& =\mathcal{U} \circ \mathcal{P}_{X} \circ \mathcal{E}^{\dagger}\left(P_{i}\right)=\mathcal{U}\left(P_{i \boxminus 1}\right)=P_{i \boxminus 1}^{\prime} .
\end{aligned}
$$

Thus following from Lemma $13, d(X) \leq d(Y)$. By a symmetric argument, we can show that $d(Y) \leq d(X)$ as well.

Now we can easily prove the following:

Theorem 9: Let a quantum Markov chain $(\mathcal{H}, \mathcal{E})$ have two different BSCC decompositions:

$$
\begin{aligned}
\mathcal{H} & =\mathcal{B}_{0} \bigoplus \cdots \bigoplus \mathcal{B}_{n-1} \bigoplus \mathcal{T}_{\mathcal{E}} \\
& =\mathcal{B}_{0}^{\prime} \bigoplus \cdots \bigoplus \mathcal{B}_{n-1}^{\prime} \bigoplus \mathcal{T}_{\mathcal{E}}
\end{aligned}
$$

and let $d_{i}\left(\right.$ resp. $\left.d_{i}^{\prime}\right)$ be the period of $\mathcal{E}$ restricting on $\mathcal{B}_{i}\left(\right.$ resp. $\left.\mathcal{B}_{i}^{\prime}\right)$. Then

$$
\sum_{i=0}^{n-1} d_{i}=\sum_{i=0}^{n-1} d_{i}^{\prime} .
$$




\subsection{Limiting States}

In the last section, we have considered the limiting states of irreducible and aperiodic quantum Markov chains. In this section, let us study these for general quantum Markov chains which may be reducible.

Lemma 19: Let $\mathcal{G}=(\mathcal{H}, \mathcal{E})$ be a quantum Markov chain. Then the following statements are equivalent:

(1) For any $\rho \in D(\mathcal{H}), \lim _{n \rightarrow \infty} \mathcal{E}^{n}(\rho)$ exists;

(2) 1 is the only eigenvalue of $\mathcal{E}$ with magnitude one.

Proof. If 1 is the only eigenvalue with magnitude one, then $\mathcal{E}_{\phi}=\lim _{n \rightarrow \infty} \mathcal{E}^{n}$. On the other hand, if for any $\rho \in D(\mathcal{H}), \lim _{n \rightarrow \infty} \mathcal{E}^{n}(\rho)$ exists, then

$$
\mathcal{E}_{\phi}(\rho)=\lim _{n \rightarrow \infty} \mathcal{E}^{n}(\rho)=\mathcal{E}\left(\lim _{n \rightarrow \infty} \mathcal{E}^{n}(\rho)\right)=\mathcal{E}\left(\mathcal{E}_{\phi}(\rho)\right)
$$

So $\mathcal{E}\left(\mathcal{E}_{\phi}\right)=\mathcal{E}_{\phi}$. Note that the corresponding Jordan norm forms of $\mathcal{E} \circ \mathcal{E}_{\phi}$ and $\mathcal{E}_{\phi}$ are respectively

$$
\begin{aligned}
J J_{\phi} & =\sum_{k:\left|\lambda_{k}\right|=1} \lambda_{k} P_{k} \\
J_{\phi} & =\sum_{k:\left|\lambda_{k}\right|=1} P_{k} .
\end{aligned}
$$

Thus, whenever $\left|\lambda_{k}\right|=1$ it actually holds $\lambda_{k}=1$.

A special case of Lemma 19 where $\mathcal{E}$ is unital (that is, $\mathcal{E}(I)=I$ ) was proved in [45]. The following lemma further deals with the case when the limiting state is unique.

Lemma 20: For any quantum Markov chain $\mathcal{G}=(\mathcal{H}, \mathcal{E})$, the following statements are equivalent:

(1) There is a limiting state $\rho^{*}$, i.e. $\lim _{n \rightarrow \infty} \mathcal{E}^{n}(\rho)=\rho^{*}$ for all $\rho \in D(\mathcal{H})$. Especially, if $\mathcal{E}$ is unital, then $\rho^{*}=I / n$, where $\operatorname{dim}(\mathcal{H})=n$; 
(2) $\mathcal{H}$ contains a unique $\mathrm{BSCC} \mathcal{B}$ and $\left(\mathcal{B},\left.\mathcal{E}\right|_{\mathcal{B}}\right)$ is aperiodic;

(3) 1 is the only eigenvalue of $\mathcal{E}$ with magnitude one and its geometric multiplicity is 1 .

Proof. $(1) \Rightarrow(2)$ is easy. If $\mathcal{H}$ has two BSCCs, then there are two stationary states. This contradicts the uniqueness of limiting states. As limiting states must be stationary, by Lemma $12, \operatorname{supp}\left(\rho^{*}\right)=\mathcal{B}$. From Theorem $7,\left(\mathcal{B},\left.\mathcal{E}\right|_{\mathcal{B}}\right)$ is aperiodic.

$(2) \Rightarrow(3)$ As $\mathcal{E}_{\phi}=\lim _{i \rightarrow \infty} \mathcal{E}^{n_{i}}$, we see from Definition 17 that for any $\rho \in D(\mathcal{H})$, $\operatorname{supp}\left(\mathcal{E}_{\phi}(\rho)\right) \subseteq \mathcal{B}$. Note that $\left(\mathcal{B},\left.\mathcal{E}\right|_{\mathcal{B}}\right)$ is irreducible and aperiodic. Thus for any $\sigma \in D(\mathcal{B}), \lim _{n \rightarrow \infty} \mathcal{E}^{n}(\sigma)=\rho^{*}$ with $\operatorname{supp}\left(\rho^{*}\right)=\mathcal{B}$, and $\mathcal{E}_{\phi}(\sigma)=\rho^{*}$. From the fact $\mathcal{E}_{\phi} \circ \mathcal{E}_{\phi}=\mathcal{E}_{\phi}$, we have that for any $\rho \in D(\mathcal{H}), \mathcal{E}_{\phi}(\rho)=\rho^{*}$ and $\rho^{*}$ is the only stationary state of $\mathcal{E}_{\phi}$. By the definition of $\mathcal{E}_{\phi}$, the stationary states of $\mathcal{E}$ are also stationary states of $\mathcal{E}_{\phi}$, so 1 is the only eigenvalue of $\mathcal{E}$ with magnitude one and its geometric multiplicity is 1 .

$(3) \Rightarrow(1)$ Suppose 1 is the only eigenvalue with magnitude one. Then $\mathcal{E}_{\phi}=$ $\lim _{n \rightarrow \infty} \mathcal{E}^{n}$, i.e., for all $\rho \in D(\mathcal{H}), \lim _{n \rightarrow \infty} \mathcal{E}(\rho)$ exists. Furthermore, as limiting states must be stationary states and 1's geometric multiplicity is one, there is only one stationary state $\rho^{*}$ satisfying $\lim _{n \rightarrow \infty} \mathcal{E}(\rho)=\rho^{*}$ for any $\rho \in D(\mathcal{H})$, by [66, Corollary 6.5].

A quantum Markov chain containing only one BSCC is called an ergodic quantum Markov chain in [10] and the above result can also be obtained in the paper.

\subsection{Conclusion}

In this chapter, we obtained some useful characterizations of irreducibility and periodicity for quantum Markov chains. Based on them, we developed a periodic decomposition technique for irreducible quantum Markov chains, which is further combined with the BSCC and stationary coherence decompositions in the previ- 
ous literature to construct a three-level decomposition of general quantum Markov chains. This three-level decomposition provides us with a finer tool for algorithmic analysis and model-checking of quantum systems. We also established a limit theorem that gives a characterization of limiting states in a quantum Markov chain regarding periodicity, irreducibility, and eigenvalues of the super-operator. 


\section{Part IV}

Applications 
As quantum communicating systems (usually called quantum channels), quantum noises, and quantum memory cells can be characterized by a CPTP map (a super-operator) on a Hilbert space, we can model them by quantum Markov chains. In this part, we apply decompositions of quantum Markov chains developed in the last part on these topics as follows.

- Chapter 6. We give a novel characterization of the one-shot zero-error capacity of quantum channels.

- Chapter 7? We establish a structure theorem of decoherence-free subsystems of quantum systems. Then we develop an algorithm to generate a set of maximal decoherence-free subsystems for any given quantum system such that any other decoherence-free subsystem is a subspace of one of them. Furthermore, we use this structure theory in the quantum many-body system described by a family of matrix product states generated by a tensor and find a feasible way to numerically derive a basis for the tensor. Such a basis plays an important role in establishing the fundamental theorems of matrix product states.

- Chapter 8. We find an interesting new phenomenon that the purely classical memory cell can be super-activated to preserve quantum states, whereas the null memory cell can only be super-activated to encode classical information. Furthermore, necessary and sufficient conditions for this phenomenon are discovered so that the super-activation can be easily checked by examining certain eigenvalues of the quantum memory cell without computing the noiseless subsystems explicitly. In particular, it is found that entangled and separable stationary states are responsible for the super-activation of storing quantum and classical information, respectively. 


\section{Chapter 6}

\section{One-shot Zero-error Capacity of Quantum Channels}

The notion of the zero-error capacity of quantum channels is a straightforward but nontrivial generalization of the corresponding notion for an essential channel introduced by Shannon in [57]. It is defined as the least upper bound of the rates at which one can send classical messages correctly via a quantum channel [47]. As zero-error capacity is a fundamental quantity of quantum channels, it is desirable to find an efficient algorithm to compute it. However, it was proved by Beigi and Shor [6] that even computing one-shot zero-error capacity is QMA-complete [32]. Recently, Duan, Severini and Winter [20] found an upper bound of the zero-error capacity of quantum channels by introducing a notion of quantum Lovász number.

In this chapter, we give a new angle to look at the structure of quantum channels by the periodic and BSCC decompositions of quantum Markov chains developed in the last part. In particular, it gives a novel characterization of one-shot zero-error capacity for general quantum channels.

\subsection{One-shot Zero-error Capacity}

Definition 23: For a quantum channel modeled by a super-operator $\mathcal{E}$, its oneshot zero-error capacity is the maximum integer $n$ for which there exists a set of states $\left\{\rho_{0}, \cdots, \rho_{n-1}\right\}$ such that $\left\{\mathcal{E}\left(\rho_{0}\right), \cdots, \mathcal{E}\left(\rho_{n-1}\right)\right\}$ can be perfectly distinguished; that is, they are mutually orthogonal.

Let us first fix several notations: for a super-operator $\mathcal{E}$ on a Hilbert space $\mathcal{H}$, 
we write:

- $\alpha(\mathcal{E})$ for the one-shot zero-error capacity of quantum channel $\mathcal{E}$;

- $\beta(\mathcal{E})$ the sum of the periods of all BSCCs in quantum Markov chain $(\mathcal{H}, \mathcal{E})$. From Corollary 9, $\beta(\mathcal{E})$ is well-defined;

- $\gamma(\mathcal{E})$ the number of BSCCs in quantum Markov chain $(\mathcal{H}, \mathcal{E})$. The welldefinedness of $\gamma(\mathcal{E})$ comes from Lemma 9

We first show a technical lemma which is useful for our later discussion.

Lemma 21: For any super-operators $\mathcal{E}$ and $\mathcal{F}$, if $\mathcal{E} \circ \mathcal{F}=\mathcal{F}$ then $\alpha(\mathcal{F}) \leq \gamma(\mathcal{E})$.

Proof: Suppose $\left\{\rho_{i}: 1 \leq i \leq \alpha(\mathcal{F})\right\}$ is a set of states such that $\mathcal{F}\left(\rho_{i}\right)$ and $\mathcal{F}\left(\rho_{j}\right)$ are orthogonal whenever $i \neq j$. From $\mathcal{E} \circ \mathcal{F}=\mathcal{F}$, each $\mathcal{F}\left(\rho_{i}\right)$ is a fixed point state of $\mathcal{E}$, and its support contains a BSCC. Hence, $\alpha(\mathcal{F}) \leq \gamma(\mathcal{E})$.

The next lemma collects some relations between the three quantities $\alpha(\mathcal{E}), \beta(\mathcal{E})$, and $\gamma(\mathcal{E})$ for different variants $\left(\mathcal{E}_{\infty}\right.$ and $\mathcal{E}_{\phi}$ defined by Eqs. 2.1$)$ and $(2.2)$, respectively) of a super-operator $\mathcal{E}$.

Lemma 22: Let $\mathcal{E}$ be a super-operator on $\mathcal{H}$. Then

(1) $\alpha(\mathcal{E}) \geq \beta(\mathcal{E}) \geq \gamma(\mathcal{E})$

(2) $\mathcal{E}_{\phi} \circ \mathcal{E}_{\phi}=\mathcal{E}_{\phi}, \mathcal{E}_{\infty} \circ \mathcal{E}_{\infty}=\mathcal{E}_{\infty}$, and $\mathcal{E} \circ \mathcal{E}_{\infty}=\mathcal{E}_{\infty}$;

(3) $\alpha\left(\mathcal{E}_{\infty}\right)=\beta\left(\mathcal{E}_{\infty}\right)=\gamma\left(\mathcal{E}_{\infty}\right) \leq \operatorname{tr}\left(J_{\infty}\right)$;

(4) $\alpha\left(\mathcal{E}_{\phi}\right)=\beta\left(\mathcal{E}_{\phi}\right)=\gamma\left(\mathcal{E}_{\phi}\right) \leq \operatorname{tr}\left(J_{\phi}\right)$.

Proof: (1) and (2) are easy to check, and the first part of (3) and (4) follows immediately from (1), (2) and Lemma 21. 
The following example shows the two inequalities in Lemma 22 hold strictly in general.

Example 4: Consider a quantum Markov chain $\mathcal{G}=(\mathcal{H}, \mathcal{E})$ with state space $\mathcal{H}=\operatorname{lin} . \operatorname{span}\{|0\rangle, \cdots,|3\rangle\}$ and the super-operator $\mathcal{E}(\cdot)=\sum_{i=1}^{4} E_{i} \cdot E_{i}^{\dagger}$, where:

$$
\begin{aligned}
& E_{1}=\frac{1}{\sqrt{2}}(|0\rangle\langle 0+1|+| 2\rangle\langle 2+3|), \\
& E_{2}=\frac{1}{\sqrt{2}}(|0\rangle\langle 0-1|+| 2\rangle\langle 2-3|), \\
& E_{3}=\frac{1}{\sqrt{2}}(|1\rangle\langle 0+1|+| 3\rangle\langle 2+3|), \\
& E_{4}=\frac{1}{\sqrt{2}}(|1\rangle\langle 0-1|+| 3\rangle\langle 2-3|),
\end{aligned}
$$

$|0 \pm 1\rangle=(|0\rangle \pm|1\rangle) / \sqrt{2},|2 \pm 3\rangle=(|2\rangle \pm|3\rangle) / \sqrt{2}$. It is easy to see that the matrix representation of $\mathcal{E}$ is diagonalizable and, 1 and 0 are the only eigenvalues of $\mathcal{E}$ with algebraic multiplicities being 4 and 12 , respectively. So $\mathcal{E}=\mathcal{E}_{\infty}=\mathcal{E}_{\phi}$ and $\operatorname{tr}\left(J_{\phi}\right)=\operatorname{tr}\left(J_{\infty}\right)=4$. Moreover, we can easily obtain that $\alpha(\mathcal{E})=2$, so $\alpha\left(\mathcal{E}_{\infty}\right)=\alpha\left(\mathcal{E}_{\phi}\right)=2$.

A more interesting relation between $\alpha(\mathcal{E}), \beta(\mathcal{E}), \gamma(\mathcal{E})$ is established in the following theorem:

Theorem 10: Let $\mathcal{E}$ be a super-operator on $\mathcal{H}$. Then

$$
\alpha\left(\mathcal{E}_{\infty}\right)=\gamma(\mathcal{E}) \text { and } \alpha\left(\mathcal{E}_{\phi}\right)=\beta(\mathcal{E})
$$

Proof: Note that $\gamma(\mathcal{E}) \leq \frac{1}{n} \sum_{i=1}^{n} \alpha\left(\mathcal{E}^{i}\right)$ for any $n \geq 1$. Thus, $\gamma(\mathcal{E}) \leq \alpha\left(\mathcal{E}_{\infty}\right)$. The reverse inequality follows from Lemma $22(2)$ and Lemma 21. So, $\alpha\left(\mathcal{E}_{\infty}\right)=\gamma(\mathcal{E})$.

Let $\mathcal{H}=\bigoplus_{i=0}^{n-1} \mathcal{B}_{i} \oplus \mathcal{T}_{\mathcal{E}}$ be the BSCC decomposition of $\mathcal{H}$ according to $\mathcal{E}$ where $\mathcal{T}_{\mathcal{E}}$ is the largest transient subspace. Furthermore, by the periodic decomposition, each $\mathcal{B}_{i}$ can be decomposed into $d_{i}$ orthogonal subspaces $\mathcal{B}_{i}=\bigoplus_{j=0}^{d_{i}-1} \mathcal{B}_{i, j}$ such that for each $j, \mathcal{E}\left(\mathcal{B}_{i, j}\right)=\mathcal{B}_{i, j \boxplus 1}$ where $\boxplus$ denotes addition modulo $d_{i}$. Then we have: 
Claim: for any $0 \leq i \leq n-1$ and $0 \leq j \leq d_{i}-1, \mathcal{B}_{i, j}$ is a BSCC of $\mathcal{E}_{\phi}$.

It is easy to see that $\mathcal{B}_{i}$ is an invariant subspace under $\mathcal{E}_{\phi}$, and by [66, Theorem 6.6], for any $\rho \in D\left(\mathcal{B}_{i}\right),\left.\mathcal{E}_{\phi}\right|_{\mathcal{B}_{i}}(\rho)=\lim _{n \rightarrow \infty} \mathcal{E}^{d_{i} n}(\rho)$. Then by Theorem 8 , $\left(\mathcal{B}_{i, j},\left.\mathcal{E}^{d_{i}}\right|_{\mathcal{B}_{i, j}}\right)$ is irreducible and aperiodic, so $\mathcal{B}_{i, j}$ is also an invariant subspace under $\mathcal{E}_{\phi}$. By invoking Theorem 7, we obtain that for any $\rho \in D\left(\mathcal{B}_{i, j}\right)$

$$
\mathcal{E}_{\phi}(\rho)=\lim _{n \rightarrow \infty} \mathcal{E}^{d_{i} n}(\rho)=\rho_{i, j}^{*}
$$

where $\rho_{i, j}^{*}$ is the limiting state for $\left(\mathcal{B}_{i, j},\left.\mathcal{E}^{d_{i}}\right|_{\mathcal{B}_{i, j}}\right)$ and $\operatorname{supp}\left(\rho_{i, j}^{*}\right)=\mathcal{B}_{i, j}$. Therefore, $\rho_{i, j}^{*}$ is the unique stationary state in $D\left(\mathcal{B}_{i, j}\right)$ and $\mathcal{B}_{i, j}$ is a BSCC of $\mathcal{E}_{\phi}$, following Lemma 8 .

With this claim and noting that $\mathcal{T}_{\mathcal{E}}$ is also the transient subspace of $\mathcal{E}_{\phi}$, we immediately know that $\gamma\left(\mathcal{E}_{\phi}\right)=\beta(\mathcal{E})$. Then $\alpha\left(\mathcal{E}_{\phi}\right)=\beta(\mathcal{E})$ follows from Lemma 22 (4).

By the above theorem, a way to check the uniqueness of the BSCC and periodic decompositions can be obtained.

Lemma 23: Let $\mathcal{E}$ be a super-operator on $\mathcal{H}$. Then

(1) $\gamma(\mathcal{E})=\operatorname{tr}\left(J_{\infty}\right)$ if and only if the BSCC decomposition of $\mathcal{E}$ is unique;

(2) $\beta(\mathcal{E})=\operatorname{tr}\left(J_{\phi}\right)$ if and only if the periodic decomposition of $\mathcal{E}$ is unique.

Proof. It is easy to see that $\gamma(\mathcal{E})=\operatorname{tr}\left(J_{\infty}\right)$ if and only if there are no stationary coherences between the BSCCs of $\mathcal{E}$. Also by Theorem 4, the BSCC decomposition of $\mathcal{E}$ is unique if and only if there are no stationary coherences between the BSCCs of $\mathcal{E}$. By the same argument and noting from the proof of Theorem 10 that the periodic decomposition of $\mathcal{E}$ coincides with the BSCC decomposition of $\mathcal{E}_{\phi}$, we derive that $\gamma\left(\mathcal{E}_{\phi}\right)=\operatorname{tr}\left(J_{\phi}\right)$ if and only if the periodic decomposition of $\mathcal{E}$ is unique. Then we complete the proof by noting $\gamma\left(\mathcal{E}_{\phi}\right)=\beta(\mathcal{E})$. 
As a consequence of Lemma 23, combining with Theorem 17 and [66, Theorem 6.6], we can claim that the periodic decomposition of irreducible quantum Markov chains is unique.

Furthermore, we consider the limit of one-shot zero-error capacity of the composition of multiple copies of channel $\mathcal{E}$.

Theorem 11: Let $\mathcal{E}$ be a super-operator on $\mathcal{H}$. Then

$$
\inf _{n} \alpha\left(\mathcal{E}^{n}\right)=\lim _{n \rightarrow \infty} \alpha\left(\mathcal{E}^{n}\right)=\beta(\mathcal{E})
$$

Proof: We first note that $\left\{\alpha\left(\mathcal{E}^{n}\right)\right\}$ is a decreasing sequence and $\alpha\left(\mathcal{E}^{n}\right) \geq 1$ for all $n \geq 1$. So, $\lim _{n \rightarrow \infty} \alpha\left(\mathcal{E}^{n}\right)$ exists and $\inf _{n} \alpha\left(\mathcal{E}^{n}\right)=\lim _{n \rightarrow \infty} \alpha\left(\mathcal{E}^{n}\right)$. By Lemma 3, there exists an increasing sequence $\left\{n_{i}\right\}$ such that $\mathcal{E}_{\phi}=\lim _{i \rightarrow \infty} \mathcal{E}^{n_{i}}$. Thus $\lim _{i \rightarrow \infty} \alpha\left(\mathcal{E}^{n_{i}}\right)=$ $\alpha\left(\mathcal{E}_{\phi}\right)$ and $\alpha\left(\mathcal{E}_{\phi}\right)=\beta(\mathcal{E})$, and finally we have $\lim _{n \rightarrow \infty} \alpha\left(\mathcal{E}^{n}\right)=\beta(\mathcal{E})$.

The significance of Theorem 11 can be better understood by considering the following scenario: Suppose Alice wants to send messages to Bob by a quantum communication over a long distance ( $\geq 1000 \mathrm{~km}$, say, from Beijing to Sydney). Due to fiber attenuation and operation errors accumulated over the entire communication distance, they can not communicate with each other directly. Alice must first send the message to some quantum repeater, and then the repeater resends it to the next one. After $n-1$ quantum repeaters, the message will be received by Bob. Then $\alpha\left(\mathcal{E}^{n}\right)$ is the zero-error capacity of this communication process. Theorem 11 shows that provided $n$ is large enough, $\beta(\mathcal{E})$ can be accepted as a good approximation of $\alpha\left(\mathcal{E}^{n}\right)$.

It was shown in [20] that one-shot zero-error capacity of channel $\mathcal{E}$ with Kraus operators $\left\{E_{i}\right\}$ only depends on the so-called non-commutative confusability graph defined as lin.span $\left\{E_{i}^{\dagger} E_{j}: i, j\right\}$. By Theorems 10 and 11, we see that $\lim _{n \rightarrow \infty} \alpha\left(\mathcal{E}^{n}\right)$ only depends on the non-commutative confusability graph of $\mathcal{E}_{\phi}$. 


\subsection{A New Characterization}

In this section, we give a new characterization of one-shot zero-error capacity of quantum channels by $\beta(\cdot)$ and $\gamma(\cdot)$. For this purpose, we recall from [8] that transpose channels of $\mathcal{E}$ is defined to be

$$
\mathcal{R}_{P_{\mathcal{X}}}=\Pi \circ \mathcal{E}^{\dagger} \circ \mathcal{N}
$$

where $\mathcal{X}$ is a subspace of $\mathcal{H}, P_{\mathcal{X}}$ is the projector onto $\mathcal{X}$, and $\mathcal{N}$ is a normalization map. That is, if the Kraus operators of $\mathcal{E}$ are $\left\{E_{i}\right\}$, then

$$
\begin{aligned}
\Pi(\rho) & =P_{\mathcal{X}} \rho P_{\mathcal{X}}, \\
\mathcal{E}^{\dagger}(\rho) & =\sum_{i} E_{i}^{\dagger} \rho E_{i}, \\
\mathcal{N}(\rho) & =\mathcal{E}\left(P_{\mathcal{X}}\right)^{-\frac{1}{2}} \rho \mathcal{E}\left(P_{\mathcal{X}}\right)^{-\frac{1}{2}} .
\end{aligned}
$$

Note that the inverse in $\mathcal{E}\left(P_{\mathcal{X}}\right)^{-\frac{1}{2}}$ is taken on the support of $\mathcal{E}\left(P_{\mathcal{X}}\right)$. In general, $\mathcal{R}_{P_{\mathcal{X}}}$ is not trace-preserving, but on the subspace $\mathcal{E}(\mathcal{X}), \mathcal{R}_{P_{\mathcal{X}}}$ is a CPTP map. Furthermore, $\mathcal{R}_{P_{\mathcal{X}}} \circ \mathcal{E}$ is a CPTP map on the subspace $\mathcal{X}$, and $\mathcal{X}$ is an invariant subspace in the sense that $\mathcal{R}_{P_{\mathcal{X}}} \circ \mathcal{E}(\mathcal{X}) \subseteq \mathcal{X}$

Theorem 12: Let $\mathcal{E}$ be a super-operator on $\mathcal{H}$. Then

$$
\alpha(\mathcal{E})=\max _{\mathcal{X} \subseteq \mathcal{H}} \gamma_{\mathcal{X}}\left(\mathcal{R}_{P_{\mathcal{X}}} \circ \mathcal{E}\right)=\max _{\mathcal{X} \subseteq \mathcal{H}} \beta_{\mathcal{X}}\left(\mathcal{R}_{P_{\mathcal{X}}} \circ \mathcal{E}\right)
$$

To prove Theorem 12 , we need the following technical lemma.

Lemma 24: Let $\mathcal{E}$ be a super-operator on $\mathcal{H}$ and $\mathcal{X}$ an subspace of $\mathcal{H}$. Then

$$
\alpha(\mathcal{E}) \geq \alpha\left(\mathcal{R}_{P_{\mathcal{X}}} \circ \mathcal{E}\right)
$$

Proof: $\mathcal{R}_{P_{\mathcal{X}}}$ and $\mathcal{E}$ satisfy the assumption of Lemma 5, so for any states $\rho, \sigma \in$ $D(\mathcal{H})$, we have:

$$
\|\rho-\sigma\|_{1} \geq\|\mathcal{E}(\rho-\sigma)\|_{1} \geq\left\|\mathcal{R}_{P_{\mathcal{X}}} \circ \mathcal{E}(\rho-\sigma)\right\|_{1}
$$


By the above inequality and the definition of $\alpha(\mathcal{E})$, we conclude that $\alpha(\mathcal{E}) \geq \alpha\left(\mathcal{R}_{P_{\mathcal{X}}}\right.$ ○ $\mathcal{E})$

Now we are ready to prove Theorem 12 .

Proof: First, using Lemmas 22 and 24 , for any subspace $\mathcal{X}$ of $\mathcal{H}$, we obtain:

$$
\alpha(\mathcal{E}) \geq \alpha\left(\mathcal{R}_{P_{\mathcal{X}}} \circ \mathcal{E}\right) \geq \beta_{\mathcal{X}}\left(\mathcal{R}_{P_{\mathcal{X}}} \circ \mathcal{E}\right) \geq \gamma_{\mathcal{X}}\left(\mathcal{R}_{P_{\mathcal{X}}} \circ \mathcal{E}\right)
$$

Let $n=\alpha(\mathcal{E})$. This means that there are $n$ orthogonal states $\left\{\rho_{i}\right\}_{i=1}^{n}$ such that $\left\{\mathcal{E}\left(\rho_{i}\right)\right\}_{i=1}^{n}$ are still mutually orthogonal. Let $C$ be the convex set generated by $\left\{\rho_{i}\right\}_{i=1}^{n}$ and $P$ the projector onto the support space of $C$. For any $\rho=\sum_{i=1}^{n} p_{i} \rho_{i}$ and $\sigma=\sum_{i=1}^{n} q_{i} \rho_{i}$ in $C$, it is easy to check that for any $x \geq 0$,

$$
\begin{aligned}
& \|\mathcal{E}(\rho-x \sigma)\|_{1} \\
= & \left\|\sum_{i=1}^{n}\left(p_{i}-x q_{i}\right) \mathcal{E}\left(\rho_{i}\right)\right\|_{1} \\
= & \sum_{i=1}^{n}\left|p_{i}-x q_{i}\right| \\
= & \|\rho-x \sigma\|_{1}
\end{aligned}
$$

and hence $C$ is preserved by $\mathcal{E}$ in the sense of [8]. By [8, Theorems 1 and 2], $C$ is isometric to a subset of the fixed states of $\mathcal{R}_{P_{\mathcal{X}}} \circ \mathcal{E}$. Thus

$$
\alpha(\mathcal{E}) \leq \gamma_{\mathcal{X}}\left(\mathcal{R}_{P_{\mathcal{X}}} \circ \mathcal{E}\right)
$$

and we complete the proof by noting Eq.(6.1).

\subsection{Conclusion}

In this chapter, we found an interesting connection between one-shot zero-error capacity of quantum channels and decompositions of quantum Markov chains.

For future studies, an immediate topic is to generalize our results on the one-shot zero-error capacity to the case of $n$-shot zero-error capacity. Treating a quantum 
channel together with its state Hilbert space as a quantum generalization of Markov chain offers a way to re-examine some fundamental problems in quantum information theory, e.g., super-activation of quantum channels [12, 15]. 


\section{Chapter 7}

\section{The Structure of Decoherence-free Subsystems}

To build large-scale quantum computers, the obstacles, such as decoherence and noise, must be managed and overcome [49]. One of the effective methods for this purpose is through decoherence-free subspaces proposed by Daniel A. Lidar in [43]. A subspace of the system Hilbert space is said to be decoherence-free if the effect of the noise on it is simply unitary, and thus easily correctable. For this sake, decoherencefree subspaces are essential tools in quantum computing, where coherent control of quantum systems is the goal[42]. On the other hand, decoherence-free subspaces can be characterized as a particular case of quantum error-correcting codes to preserve quantum information from noise [42]. Indeed, we do not even need to restrict the decoherence-free dynamics to a subspace. E. Knill, R. Laflamme, and L. Viola introduced the concept of noiseless subsystems, by extending higher-dimensional irreducible representations of the algebra generating the dynamical symmetry in the system-environment interaction [34]. A subsystem is a factor in the tensor product decomposition of a subspace, and the noiseless subsystem requires the evolution on it to be identity.

Noiseless subsystems have been fully characterized in [13, 9, 7, 37, 35]. Remarkably, a structure theory of noiseless subsystems was established in [13], leading to an algorithm which finds all noiseless subsystems for a given quantum operation (i.e., the evolution of an open quantum system, mathematically modeled by a super-operator) [33, 63]. For the more general case of decoherence-free subsystems, however, a structure theory is still lacking, although several conditions for their ex- 
istence were found in [56], and subsystems with significantly reduced noises were carefully examined in [64]. Without such a structure theory, it is hard to compute all decoherence-free subsystems (subspaces) or the highest-dimensional ones for a given super-operator.

This chapter aims to develop a structure theory that shows precisely how a super-operator determines its decoherence-free subsystems, with the structure theory of noiseless subsystems as a particular case. As an application, we develop an algorithm to generate a set of maximal decoherence-free subsystems for any given super-operator such that any other decoherence-free subsystem is a subspace of one of them. Furthermore, we use this structure theory in the quantum many-body system described by a family of matrix product states generated by a tensor and find a feasible way to numerically derive a basis for the tensor. Such a basis plays a vital role in establishing the fundamental theorems of matrix product states [14, 17].

All the results in this chapter are based on our paper [26].

\subsection{Continuous Coherence}

Recall that given a quantum system $S$ with the associated (finite-dimensional) state Hilbert space $\mathcal{H}$, the evolution of the system can be mathematically modeled by a super-operator $\mathcal{E}$ on $\mathcal{H}$. We say that a quantum system $A$ is a subsystem of $S$ if $\mathcal{H}=\left(\mathcal{B}_{A} \otimes \mathcal{B}_{B}\right) \oplus\left(\mathcal{B}_{A} \otimes \mathcal{B}_{B}\right)^{\perp}$ for some co-subsystem $B$, where $\mathcal{B}_{A}$ and $\mathcal{B}_{B}$ are the state spaces of $A$ and $B$, respectively. Generally, co-subsystem $B$ is not unique, and one may construct other co-subsystems of $A$ as subspaces of $\mathcal{B}_{B}$, or by combining co-subsystems of $A$ with orthogonal supports.

Definition 24: Let $\mathcal{E}$ be a super-operator on $\mathcal{H}$. A subsystem $\mathcal{B}_{A}$ of $\mathcal{H}$ is called decoherence-free if there is a co-subsystem $\mathcal{B}_{B}$ of $\mathcal{B}_{A}$ (that is, $\mathcal{B}_{A} \otimes \mathcal{B}_{B}$ is a subspace 
of $\mathcal{H})$ and a unitary matrix $U_{A}$ on $\mathcal{B}_{A}$ such that $\forall \rho_{A} \in D\left(\mathcal{B}_{A}\right), \forall \rho_{B} \in D\left(\mathcal{B}_{B}\right)$,

$$
\exists \sigma_{B} \in D\left(\mathcal{B}_{B}\right): \mathcal{E}\left(\rho_{A} \otimes \rho_{B}\right)=U_{A} \rho_{A} U_{A}^{\dagger} \otimes \sigma_{B}
$$

Furthermore, if $U_{A}=I_{A}$, the identity operator on $\mathcal{B}_{A}$, then we say that $\mathcal{B}_{A}$ is noiseless.

Co-Subsystem $\mathcal{B}_{B}$ of decoherence-free subsystem $\mathcal{B}_{A}$ is inessential as $\mathcal{B}_{B}$ can be traced over. Noiseless subsystems have been intensely studied in the areas of quantum error correction [36, 7, 13, 35] and quantum memory [38], and can be characterized by the set of fixed points of $\mathcal{E}$. From Theorem 5, with an appropriately orthogonal decomposition of the Hilbert space $\mathcal{H}=\bigoplus_{k=1}^{n}\left(\mathcal{B}_{A_{k}} \otimes \mathcal{B}_{B_{k}}\right) \oplus \mathcal{T}_{\mathcal{E}}, \operatorname{fix}(\mathcal{E})$ admits a useful structure:

$$
\operatorname{fix}(\mathcal{E})=\bigoplus_{k=1}^{n}\left(\mathcal{L}\left(\mathcal{B}_{A_{k}}\right) \otimes \sigma_{k}\right) \oplus 0_{\mathcal{T}_{\mathcal{E}}}
$$

where $\sigma_{k}$ is a full-rank quantum state on $\mathcal{B}_{B_{k}}$. It is easy to see that for each $k$, $\mathcal{B}_{A_{k}}$ is a noiseless subsystem. Conversely, this decomposition captures all noiseless subsystems; that is, $\mathcal{B}_{A}$ is a noiseless subsystem if and only if $\mathcal{B}_{A} \subseteq \mathcal{B}_{A_{k}}$ for some $k$.

Example 5: Given $\mathcal{H}=\mathcal{B}_{A} \otimes \mathcal{B}_{B}$, and $\left\{|k\rangle_{A}\right\}_{k=0}^{3}$ and $\left\{|k\rangle_{B}\right\}_{k=0}^{2}$ are orthonormal bases of $\mathcal{B}_{A}$ and $\mathcal{B}_{B}$, respectively, let $\mathcal{E}$ be a super-operator on $\mathcal{H}$ with the Kraus operators:

$$
\begin{aligned}
& E_{1}=|00\rangle\langle 01|+| 10\rangle\langle 11|-| 20\rangle\langle 21|-| 30\rangle\langle 31| \\
& E_{2}=|01\rangle\langle 00|+| 11\rangle\langle 10|-| 21\rangle\langle 20|-| 31\rangle\langle 30| \\
& E_{3}=|00\rangle\langle 02|+| 10\rangle\langle 12|-| 20\rangle\langle 22|-| 30\rangle\langle 32|
\end{aligned}
$$

where $|k l\rangle=|k\rangle_{A} \otimes|l\rangle_{B}$. It is easy to calculate the fixed-point decomposition of $\mathcal{H}$ as

$$
\mathcal{H}=\bigoplus_{l=1}^{2}\left[\mathcal{B}_{l} \otimes \mathcal{H}^{\prime}\right] \oplus \mathcal{K}
$$


where $\mathcal{B}_{1}=$ lin.span $\left\{|0\rangle_{A},|1\rangle_{A}\right\}, \mathcal{B}_{2}=\operatorname{lin} . \operatorname{span}\left\{|2\rangle_{A},|3\rangle_{A}\right\}, \mathcal{H}^{\prime}=\operatorname{lin} . \operatorname{span}\left\{|0\rangle_{B},|1\rangle_{B}\right\}$, and $\mathcal{K}=\mathcal{B}_{A} \otimes \operatorname{lin} . \operatorname{span}\left\{|2\rangle_{B}\right\}$. Then we can store 1-qubit quantum information in $\mathcal{B}_{1}$ or $\mathcal{B}_{2}$

Given a super-operator $\mathcal{E}$ on $\mathcal{H}$, applying BSCC decomposition, we can decompose $\mathcal{H}$ into a set of mutually orthogonal BSCCs with the largest transient subspace $\mathcal{T}_{\mathcal{E}}:$

$$
\mathcal{H}=\bigoplus_{p=1}^{m} \mathcal{B}_{p} \oplus \mathcal{T}_{\mathcal{E}}
$$

It is worth noting that each $\mathcal{B}_{p}$ is invariant under $\mathcal{E}$, i.e. for any $A \in \mathcal{L}\left(\mathcal{B}_{p}\right)$, $\mathcal{E}(A) \in \mathcal{L}\left(\mathcal{B}_{p}\right)$. Thus, the Kraus operators $\left\{E_{k}\right\}$ of $\mathcal{E}$ have the corresponding block form:

$$
E_{k}=\left[\begin{array}{cccc|c}
E_{k, 1} & & & & \\
& E_{k, 2} & & & T_{k} \\
& & \ddots & & \\
& & E_{k, m} & \\
\hline & & & K_{k}
\end{array}\right]
$$

for some operators $E_{k, p} \in \mathcal{L}\left(\mathcal{B}_{p}\right), K_{k} \in \mathcal{L}\left(\mathcal{T}_{\mathcal{E}}\right)$, and $T_{k} \in \mathcal{L}\left(\mathcal{T}_{\mathcal{E}}, \mathcal{T}_{\mathcal{E}}^{\perp}\right)$. We then define a set of associated maps $\left\{\mathcal{E}_{p, q}: p, q=1, \ldots, m\right\}$ of $\mathcal{E}$ :

$$
\mathcal{E}_{p, q}(\cdot)=\sum_{k} E_{k, p} \cdot E_{k, q}^{\dagger}
$$

Obviously, for any $p$ and $q, \mathcal{E}_{p, q}$ is a linear map from $\mathcal{L}\left(\mathcal{B}_{q}, \mathcal{B}_{p}\right)$ to itself. If $p \neq q$, $\mathcal{L}\left(\mathcal{B}_{q}, \mathcal{B}_{p}\right)$ can be viewed as (outer) coherences from $\mathcal{B}_{q}$ to $\mathcal{B}_{p}$, i.e. upper off-diagonal blocks of all matrices restricted in the decomposition $\mathcal{B}_{p} \oplus \mathcal{B}_{q}$. Thus the coherence between $\mathcal{B}_{p}$ and $\mathcal{B}_{q}$ is $\mathcal{L}\left(\mathcal{B}_{q}, \mathcal{B}_{p}\right) \oplus \mathcal{L}\left(\mathcal{B}_{p}, \mathcal{B}_{q}\right)$ and $\mathcal{L}\left(\mathcal{B}_{q}\right)$ can be regarded as inner coherences.

For all $p$ and $q$, the following two properties are easy to observe: 
(1) $\mathcal{L}\left(\mathcal{B}_{q}, \mathcal{B}_{p}\right)$ is invariant under $\mathcal{E}$; that is, for all $A \in \mathcal{L}\left(\mathcal{B}_{q}, \mathcal{B}_{p}\right), \mathcal{E}(A) \in \mathcal{L}\left(\mathcal{B}_{q}, \mathcal{B}_{p}\right)$;

(2) $\lambda\left(\mathcal{E}_{p, q}\right) \subseteq \lambda(\mathcal{E})$, where recall that $\lambda(\mathcal{E})$ is the set of all eigenvalues of $\mathcal{E}$.

Furthermore, the coherence $\mathcal{L}\left(\mathcal{B}_{q}, \mathcal{B}_{p}\right)$ is said to be continuous if there exists $A \in \mathcal{L}\left(\mathcal{B}_{q}, \mathcal{B}_{p}\right)$ such that $\mathcal{E}(A)=\mathcal{E}_{p, q}(A)=e^{i \theta} A$ for some real number $\theta$; that is, $\lambda\left(\mathcal{E}_{p, q}\right)$ has an element with magnitude one. Specially, if $\theta=0$, then $\mathcal{L}\left(\mathcal{B}_{q}, \mathcal{B}_{p}\right)$ is stationary. Obviously, inner coherence $\mathcal{L}\left(\mathcal{B}_{q}\right)$ is always stationary because a superoperator has at least one stationary state. Stationary coherences have been introduced in Chapter 4 and intensely studied in [5].

\subsection{Structure Theorem}

By the definition, a decoherence-free subsystem $\mathcal{B}_{A}$ is a small section of the whole state space $\mathcal{H}$ in which the effect of the quantum noise modeled by $\mathcal{E}$ is equivalent to a unitary transformation. From Eq.(7.1), the restriction of $\mathcal{E}$ onto $\mathcal{B}_{A} \otimes \mathcal{B}_{B}$, where $\mathcal{B}_{B}$ is the co-subsystem of $\mathcal{B}_{A}$, can be written as

$$
\mathcal{E}_{A B}=\mathcal{U}_{A} \otimes \mathcal{E}_{B}
$$

where $\mathcal{U}_{A}$ is a unitary super-operator on $\mathcal{B}_{A}$ and $\mathcal{E}_{B}$ is a super-operator on $\mathcal{B}_{B}$. By the BSCC decomposition, $\mathcal{B}_{B}$ can be chosen to be irreducible. From now on, we assume without loss of generality that the co-subsystem of a decoherence-free subsystem is always irreducible.

First, we observe that the joint system of a decoherence-free subsystem and its irreducible co-subsystem can be decomposed into BSCCs with continuous coherences.

Theorem 13: Given a super-operator $\mathcal{E}$ on

$$
\mathcal{H}=\left(\mathcal{B}_{A} \otimes \mathcal{B}_{B}\right) \oplus\left(\mathcal{B}_{A} \otimes \mathcal{B}_{B}\right)^{\perp}
$$


Let $\mathcal{B}_{A}$ be a decoherence-free subsystem and $U_{A}$ the corresponding unitary matrix in Eq. (7.1). If $\{|x\rangle\}_{x=1}^{m}$ is a set of mutually orthogonal eigenvectors of $U_{A}$ and $\mathcal{B}_{x}=\operatorname{lin} . \operatorname{span}\{|x\rangle\} \otimes \mathcal{B}_{B}$, then for all $1 \leq p, q \leq m, \mathcal{B}_{p}$ is a BSCC and $\mathcal{L}\left(\mathcal{B}_{p}, \mathcal{B}_{q}\right)$ is continuous.

Proof. Note that we assume $\mathcal{B}_{B}$ is irreducible. Let $\rho$ be the unique stationary state of $\mathcal{E}_{B}$. Then for any $x,|x\rangle\langle x| \otimes \rho$ is a minimal stationary state of $\mathcal{E}$, and hence $\mathcal{B}_{x}$ is minimal. Furthermore, note that $U_{A}|x\rangle=e^{i \theta_{x}}|x\rangle$ for some $\theta_{x}$. Thus

$$
\mathcal{E}(|p\rangle\langle q| \otimes \rho)=e^{i\left(\theta_{p}-\theta_{q}\right)}|p\rangle\langle q| \otimes \rho
$$

for all $p$ and $q$.

Theorem 13 indicates that BSCCs with continuous coherences play an essential role in determining decoherence-free subsystems. To check if two orthogonal BSCCs have a continuous coherence, we present the following lemma.

Lemma 25: Let $\mathcal{E}$ be a super-operator on $\mathcal{H}$ with the orthogonal decomposition presented in Eq. (7.3). Then for any $1 \leq p, q \leq m, \mathcal{L}\left(\mathcal{B}_{p}, \mathcal{B}_{q}\right)$ is continuous if and only if there is a unitary matrix $U$ and a real number $\theta$ such that $E_{k, p}=e^{i \theta} U E_{k, q} U^{\dagger}$ for all $k$. Furthermore, $\mathcal{B}_{p} \simeq \mathcal{B}_{q}$.

Proof. Assume that $\mathcal{L}\left(\mathcal{B}_{p}, \mathcal{B}_{q}\right)$ is continuous; that is, there is a matrix $A \in \mathcal{L}\left(\mathcal{B}_{p}, \mathcal{B}_{q}\right)$ such that $\mathcal{E}(A)=e^{i \theta} A$ for some real number $\theta$. Let $V=e^{-i \theta} P_{q}+I-P_{q}$, where $P_{x}$ is the projector onto $\mathcal{B}_{x}$, and $\mathcal{V} \circ \mathcal{E}(A)=A$ with $\mathcal{V}(\cdot)=V \cdot V^{\dagger}$. Moreover, it is obvious that $\mathcal{B}_{p}$ and $\mathcal{B}_{q}$ are also orthogonal BSCCs under $\mathcal{V} \circ \mathcal{E}$ by the decomposition Eq. (7.3). Therefore, there is a stationary coherence from $\mathcal{B}_{q}$ to $\mathcal{B}_{p}$ under $\mathcal{V} \circ \mathcal{E}$. From Lemma 11 , we have $\mathcal{B}_{p} \simeq \mathcal{B}_{q}$, and there exists some unitary matrix $U$ such that for any $k$,

$$
E_{k, p}=e^{i \theta} U E_{k, q} U^{\dagger} .
$$


Conversely, for any $p$ and $q$, let

$$
\mathcal{E}_{q, p}(\cdot)=\sum_{k} E_{k, q} \cdot E_{k, p}^{\dagger}=\sum_{k} e^{-i \theta} E_{k, q} \cdot U E_{k, q}^{\dagger} U^{\dagger}
$$

Its matrix representation reads

$$
\begin{aligned}
M_{q, p} & =\sum_{k} e^{-i \theta} E_{k, q} \otimes\left(U E_{k, q} U^{\dagger}\right)^{*} \\
& =e^{-i \theta}\left(I \otimes U^{*}\right)\left(\sum_{k} E_{k, q} \otimes E_{k, q}^{*}\right)\left(I \otimes{U^{*}}^{\dagger}\right) .
\end{aligned}
$$

As $\lambda\left(M_{q, p}\right)=\lambda\left(\mathcal{E}_{q, p}\right)$ and $\sum_{k} E_{k, q} \otimes E_{k, q}^{*}$ is the matrix representation of $\mathcal{E}_{q, q}$ which is a super-operator and has 1 as one of its eigenvalues, we have $e^{-i \theta} \in \lambda\left(\mathcal{E}_{q, p}\right)$.

Corollary 3: Let $\mathcal{E}$ be a super-operator on $\mathcal{H}$ with the orthogonal decomposition presented in Eq. (7.3). Then the relation $\left\{(p, q): 1 \leq p, q \leq m, \mathcal{L}\left(\mathcal{B}_{p}, \mathcal{B}_{q}\right)\right.$ is continuous $\}$ is an equivalence relation. That is, for any $p, q$, and $r$,

(1) (reflexivity) $\mathcal{L}\left(\mathcal{B}_{p}, \mathcal{B}_{p}\right)$ is continuous;

(2) (symmetry) if $\mathcal{L}\left(\mathcal{B}_{p}, \mathcal{B}_{q}\right)$ is continuous, then so is $\mathcal{L}\left(\mathcal{B}_{q}, \mathcal{B}_{p}\right)$;

(3) (transitivity) if $\mathcal{L}\left(\mathcal{B}_{p}, \mathcal{B}_{q}\right)$ and $\mathcal{L}\left(\mathcal{B}_{q}, \mathcal{B}_{r}\right)$ are both continuous, then so is $\mathcal{L}\left(\mathcal{B}_{p}, \mathcal{B}_{r}\right)$.

With Corollary $3, \mathcal{L}\left(\mathcal{B}_{p}, \mathcal{B}_{q}\right)$ is continuous coherence if and only if so is $\mathcal{L}\left(\mathcal{B}_{q}, \mathcal{B}_{p}\right)$. Thus in the following, we simply say that there is a continuous coherence between $\mathcal{B}_{p}$ and $\mathcal{B}_{q}$ without referring to the direction. Then we group together BSCCs by continuous coherences.

Theorem 14: Let $\mathcal{E}$ be a super-operator on $\mathcal{H}$. There is a unique orthogonal decomposition of $\mathcal{H}$

$$
\mathcal{H}=\bigoplus_{l=1}^{L} \mathcal{X}_{l} \oplus \mathcal{T}_{\mathcal{E}}
$$

where 
(1) each $\mathcal{X}_{l}$ is either a BSCC or can be further decomposed into mutually orthogonal BSCCs with continuous coherences between any two of them:

$$
\mathcal{X}_{l}=\bigoplus_{p=1}^{m_{l}} \mathcal{B}_{l, p} \simeq \mathbb{C}^{m_{l}} \otimes \mathcal{B}_{l}, \quad \mathcal{B}_{l} \simeq \mathcal{B}_{l, p} \quad \forall p
$$

such that the Kraus operators $\left\{E_{k}\right\}$ of $\mathcal{E}$ have a corresponding block form:

$$
E_{k} \simeq\left[\begin{array}{ccc|c}
U_{1} \otimes E_{k, 1} & & & \\
& \ddots & & T_{k} \\
& & & \\
& & U_{L} \otimes E_{k, L} & \\
\hline & 0 & & K_{k}
\end{array}\right]
$$

for some operators $E_{k, l} \in \mathcal{L}\left(\mathcal{B}_{l}\right), K_{k} \in \mathcal{L}\left(\mathcal{T}_{\mathcal{E}}\right), T_{k} \in \mathcal{L}\left(\mathcal{T}_{\mathcal{E}}, \mathcal{T}_{\mathcal{E}}^{\perp}\right)$, and unitary matrix $U_{l}=\operatorname{diag}\left(e^{i \theta_{l, 1}}, \cdots, e^{i \theta_{l, m_{l}}}\right)$ for some real numbers $\left\{\theta_{l, p}\right\}_{p=1}^{m_{l}}$ on $\mathbb{C}^{m_{l}}$. Moreover, $\mathcal{B}_{l}$ is irreducible under $\mathcal{E}_{l}(\cdot)=\sum_{k} E_{k, l} \cdot E_{k, l}^{\dagger}$. Furthermore,

$$
f i x(\mathcal{E})=\bigoplus_{l}\left[f i x\left(\mathcal{U}_{l}\right) \otimes \rho_{l}\right] \oplus 0_{\mathcal{T}_{\mathcal{E}}}
$$

where $\mathcal{U}_{l}(\cdot)=U_{l} \cdot U_{l}^{\dagger}$.

(2) there is no continuous coherence between any BSCCs $\mathcal{B}_{l, p}$ and $\mathcal{B}_{l^{\prime}, p^{\prime}}$ whenever $l \neq l^{\prime}$.

Proof. By the structure of noiseless subsystems, there is a unique orthogonal decomposition of $\mathcal{H}$ as

$$
\mathcal{H}=\bigoplus_{l=1}^{L^{\prime}} \mathcal{X}_{l}^{\prime} \oplus \mathcal{T}_{\mathcal{E}}
$$

such that for any orthogonal BSCCs $\mathcal{B}_{1}$ and $\mathcal{B}_{2}$, they have stationary coherences if and only if $\mathcal{B}_{1} \oplus \mathcal{B}_{2} \in \mathcal{X}_{l}^{\prime}$ for some $l$. Then we divide each $\left\{\mathcal{X}_{l}^{\prime}\right\}$ into a finite number of disjoint subsets by continuous coherences; that is for any $l_{1} \neq l_{2}$, if there is a continuous coherence between any BSCCs in $\mathcal{X}_{l_{1}}^{\prime}$ and $\mathcal{X}_{l_{2}}^{\prime}$, then they are in the same subset. This can be done as the existence of continuous coherences is an 
equivalence relation by Corollary 3 . Then we define $\left\{\mathcal{X}_{l}\right\}_{l=1}^{L}$ to be the set of the direct sum of all elements in each subset. Therefore, $\mathcal{H}$ can be uniquely decomposed as $\mathcal{H}=\bigoplus_{l} \mathcal{X}_{l} \oplus \mathcal{T}_{\mathcal{E}}$. Obviously, for any two orthogonal BSCCs $\mathcal{B}_{l_{1}} \in \mathcal{X}_{l_{1}}$ and $\mathcal{B}_{l_{2}} \in \mathcal{X}_{l_{2}}$, $\mathcal{L}\left(\mathcal{B}_{l_{1}}, \mathcal{B}_{l_{2}}\right)$ is continuous if and only if $l_{1}=l_{2}$.

Furthermore, for each $l, \mathcal{X}_{l}$ can be further decomposed into mutually orthogonal BSCCs:

$$
\mathcal{X}_{l}=\oplus_{p=1}^{m_{l}} \mathcal{B}_{l, p}
$$

By Lemma 25, in an appropriate decomposition of $\mathcal{X}_{l}=\oplus_{p=1}^{m_{l}} \mathcal{B}_{l, p} \simeq \mathbb{C}^{m_{l}} \otimes \mathcal{B}_{l}$ and $\mathcal{B}_{l} \simeq \mathcal{B}_{l, p}$ for all $p$ :

$$
E_{k}=\left[\begin{array}{ccc|c}
U_{1} \otimes E_{k, 1} & & & \\
& \ddots & & T_{k} \\
& & & \\
& & U_{L} \otimes E_{k, L} & \\
\hline & 0 & & K_{k}
\end{array}\right]
$$

and $\mathcal{B}_{l}$ is irreducible under $\mathcal{E}_{l}(\cdot):=\sum_{k} E_{k, l} \cdot E_{k, l}^{\dagger}$ for all $l$, where $U_{l}=\operatorname{diag}\left(e^{i \theta_{l, 1}}, \cdots, e^{i \theta_{l, m_{l}}}\right)$ for a set of real numbers $\left\{\theta_{l, p}\right\}_{p=1}^{m_{l}}$. From the structure of noiseless subsystems and noting that the stationary coherence is continuous, we have

$$
f i x(\mathcal{E})=\bigoplus_{l}\left[f i x\left(\mathcal{U}_{l}\right) \otimes \rho_{l}\right] \oplus 0_{\mathcal{T}_{\mathcal{E}}}
$$

where $\rho_{l}$ is the unique stationary state of $\mathcal{E}_{l}$.

Corollary 4: Let $\mathcal{E}$ be a super-operator on $\mathcal{H}$ with the unique decomposition

$$
\mathcal{H}=\bigoplus_{l}\left(\mathbb{C}^{m_{l}} \otimes \mathcal{B}_{l}\right) \oplus \mathcal{T}_{\mathcal{E}}
$$

presented in Theorem 14. For any BSCC $\mathcal{H}^{\prime}$, there is a pure state $|\psi\rangle \in \mathbb{C}^{m_{l}}$ for some $l$ such that $\mathcal{H}^{\prime}=\operatorname{lin} . \operatorname{span}\{|\psi\rangle\} \otimes \mathcal{B}_{l}$.

The above theorem shows that BSCCs with continuous coherences can be used to construct decoherence-free subsystem $\mathbb{C}^{m_{l}}$. On the other hand, we can show 
that other decoherence-free subsystems are all subspaces of the ones constructed in Eq. (7.7).

Theorem 15: Let $\mathcal{E}$ be a super-operator on $\mathcal{H}$ with the unique decomposition:

$$
\mathcal{H}=\bigoplus_{l}\left(\mathbb{C}^{m_{l}} \otimes \mathcal{B}_{l}\right) \oplus \mathcal{T}_{\mathcal{E}}
$$

presented in Theorem 14. Then a subsystem $\mathcal{B}_{A}$ is decoherence-free if and only if

(1) $\mathcal{B}_{A} \subseteq \mathbb{C}^{m_{l}}$ for some $l$, and

(2) $\mathcal{B}_{A}$ is the support of some stationary state of $\mathcal{U}_{l}(\cdot)=U_{l} \cdot U_{l}^{\dagger}$, where $U_{l}$ is the corresponding unitary matrix on $\mathbb{C}^{m_{l}}$ in the decomposition Eq. 7.8 .

Proof. Assume that $\mathcal{B}_{A}$ is decoherence-free. By Theorem 13, Theorem 14 and Corollary $4, \mathcal{B}_{A} \subseteq \mathbb{C}^{m_{l}}$ for some $l$. From the definition of decoherence-free subsystems and the fact that the restriction of $\mathcal{E}$ onto $\mathbb{C}^{m_{l}}$ is $\mathcal{U}_{l}, \mathcal{B}_{A}$ is a decoherence-free subspace under $\mathcal{U}_{l}$ and $\mathcal{U}_{l}\left(P_{A}\right)=P_{A}$, where $P_{A}$ be the projector onto $\mathcal{B}_{A}$.

To prove the opposite direction, we observe that if $\mathcal{B}_{A}$ is the support of some stationary state of $\mathcal{U}_{l}$, then $P_{A} U_{l} P_{A}=P_{A} U_{l}=U_{l} P_{A}$. Thus $\mathcal{B}_{A}$ is a decoherence-free subspace under $\mathcal{U}_{l}$. The rest of the proof is direct from Theorem 14 .

This theorem confirms that the set of decoherence-free subsystems $\left\{\mathbb{C}^{m_{l}}\right\}_{l}$ identified in Theorem 14 is optimal; that is, any other decoherence-free subsystem is a subspace of one of them. So we only need to implement the decomposition in Theorem 14, and all decoherence-free subsystems can be easily found by Theorem 15 .

One easy way of achieving this is to first transform all continuous coherences to stationary ones without changing any $\mathrm{BSCC}$, and then use the algorithm of the decomposition Eq. (7.2) for the fixed-point decomposition. To be specific, for any two operators $E_{k, p}$ and $E_{k, q}$ in Eq. (7.8) of Theorem 14, if they are unitarily equivalent with a phase $\theta$, i.e. $E_{k, p} \simeq e^{i \theta} E_{k, q}$, then let $E_{k, q}^{\prime}=e^{i \theta} E_{k, q}$ and $E_{k, p}^{\prime}=E_{k, p}$. Note 


\section{Algorithm 2 CCdecompose $(\mathcal{H}, \mathcal{E})$}

Input: A Hilbert space $\mathcal{H}$ and a super-operator $\mathcal{E}$ with Kraus operators $\left\{E_{k}\right\}_{k=1}^{d}$ on it.

Output: The two-level decomposition of $\mathcal{H}$ in the form of Eqs. (7.6) and (7.7).

Call NSDecompose $(\mathcal{H}, \mathcal{E})$ to obtain decomp. in Eqs. $4.3,4.5$

$\mathcal{L} \leftarrow\{1,2, \cdots, L\}$

for each $p$ from 1 to $L$ do

$$
\text { if } p \in \mathcal{L} \text { then }
$$

for each $q$ from $p+1$ to $L$ with $q \in \mathcal{L}$ do

$$
\begin{aligned}
& M \leftarrow \sum_{k} E_{k, p} \otimes E_{k, q}^{*} \\
& \text { if } \lambda(M) \text { has one element with magnitude one then } \\
& \eta \leftarrow \operatorname{tr}\left(E_{k, p}\right) / \operatorname{tr}\left(E_{k, q}\right) \\
& E_{k, q} \leftarrow \eta E_{k, q} \\
& \quad \mathcal{L} \leftarrow \mathcal{L} \backslash\{q\}
\end{aligned}
$$

end if

\section{end for}

\section{end if}

\section{end for}

for each $k$ from 1 to $d$ do

$$
E_{k} \leftarrow\left[\begin{array}{cc}
\bigoplus_{l=1}^{L} I_{l} \otimes E_{k, l} & T_{k} \\
0 & K_{k}
\end{array}\right]
$$

end for

return $\operatorname{SCdecompose}\left(\mathcal{H},\left\{E_{k}\right\}_{k=1}^{d}\right)$ 
that $E_{k, p}^{\prime}$ is unitarily equivalent to $E_{k, q}^{\prime}$. The continuous coherence between $\mathcal{B}_{p}$ and $\mathcal{B}_{q}$ is transformed to be stationary, and $\mathcal{B}_{p}$ and $\mathcal{B}_{q}$ are still BSCCs under the new super-operator. Using this technique, we can develop an algorithm (Algorithm 2) to implement the decomposition in Theorem 14 .

Now we return to see Example 5. By Theorem 14, we can confirm that the first subsystem $\mathcal{B}_{A} \simeq \mathbb{C}^{4}$ is decoherence-free and further show that the evolution on it is a unitary operator $|0\rangle\langle 0|+| 1\rangle\langle 1|-| 2\rangle\langle 2|-| 3\rangle\langle 3|$. Thus we can store 2-qubit information in this subsystem, which doubles the capacity of noiseless subsystems.

\subsection{Matrix Product States}

The traditional techniques for describing quantum many-body systems are usually not scalable due to the exponential growth of the dimension of the state space with the number of subsystems. Matrix Product States (MPS), a special case of tensor networks (a theoretical and numerical tool describing quantum many-body systems), have been proved to be a useful family of quantum states for the description of ground states of one-dimensional quantum many-body systems [14].

Given a tensor $\mathcal{A}=\left\{A_{k} \in \mathcal{M}_{D}\right\}_{k=1}^{d}$ with a Hilbert space $\mathcal{B}_{d}=\operatorname{lin} . \operatorname{span}\{|k\rangle\}_{k=1}^{d}$, where $\mathcal{M}_{D}$ denotes the set of all $D \times D$ complex matrices, it generates a family of translationally invariant MPS, namely

$$
V(\mathcal{A})=\left\{\left|V_{n}(\mathcal{A})\right\rangle\right\}_{n \in \mathbb{N}^{+}}
$$

where

$$
\left|V_{n}(\mathcal{A})\right\rangle=\sum_{k_{1}, \cdots, k_{n}=1}^{d} \operatorname{tr}\left(A_{k_{1}} \cdots A_{k_{n}}\right)\left|k_{1} \cdots k_{n}\right\rangle \in \mathcal{B}_{d}^{\otimes n} .
$$

Here, each $\left|V_{n}(\mathcal{A})\right\rangle$ corresponds to a state of $n$ spins of physical dimension $d$. Let the associated completely positive map be $\mathcal{E}_{\mathcal{A}}(\cdot)=\sum_{k=1}^{d} A_{k} \cdot A_{k}^{\dagger}$.

By [17], we can always find a set of irreducible tensors $\left\{\mathcal{A}_{j}\right\}_{j=1}^{m}$ with the same 
Hilbert space $\mathcal{B}_{d}$, and a set of complex number $\left\{\mu_{j}\right\}_{j=1}^{m}$ such that for any $n \in \mathbb{N}^{+}$,

$$
\left|V_{n}(\mathcal{A})\right\rangle=\sum_{j=1}^{m} \mu_{j}^{n}\left|V_{n}\left(\mathcal{A}_{j}\right)\right\rangle,
$$

where a tensor is called irreducible if the associated map is CPTP and irreducible. That is, for any tensor $\mathcal{A}$, the generated MPS can be linearly represented by MPS of a set of irreducible tensors. Furthermore, we can identify irreducible tensors that are essentially the same in the following sense.

Definition 25 ([17]): We say that two irreducible tensors $\mathcal{A}=\left\{A_{k}\right\}_{k=1}^{d}$ and $\mathcal{B}=$ $\left\{B_{k}\right\}_{k=1}^{d}$ are repeated if there exist a phase $\theta$ and a unitary matrix $U$ such that

$$
A_{k}=e^{i \theta} U B_{k} U^{\dagger}, \forall k
$$

By the definition, if $\mathcal{A}$ and $\mathcal{B}$ are repeated, then $\left|V_{n}(\mathcal{A})\right\rangle=e^{i n \theta}\left|V_{n}(\mathcal{B})\right\rangle$ for all $n \in \mathbb{N}^{+}$. Therefore, for any tensor $\mathcal{A}$, we can assume without loss of generality that the set of irreducible tensors $\left\{\mathcal{A}_{j}\right\}_{j=1}^{m}$ in Eq. (7.10) are non-repeated. Such a set is called a basis of $\mathcal{A}$.

To determine if two irreducible tensors are repeated, an obvious way is to work out the Jordan decomposition of all the matrices involved. However, as Jordan decomposition is sensitive to computational errors, it is not suitable for numerical analysis. Here we propose a more robust method to achieve this by using the results of continuous coherences.

Theorem 16: Let $\mathcal{A}=\left\{A_{k}\right\}_{k=1}^{d}$ and $\mathcal{B}=\left\{B_{k}\right\}_{k=1}^{d}$ be two irreducible tensors. Then they are repeated if and only if $\mathcal{E}_{\mathcal{A}, \mathcal{B}}$ has an eigenvalue with magnitude one, where $\mathcal{E}_{\mathcal{A}, \mathcal{B}}=\sum_{k=1}^{d} A_{k} \cdot B_{k}^{\dagger}$.

Proof. Let $A_{k} \in \mathcal{L}\left(\mathcal{B}_{\mathcal{A}}\right)$ and $B_{k} \in \mathcal{L}\left(\mathcal{B}_{\mathcal{B}}\right)$ for all $k$, and $\mathcal{E}$ be a super-operator on $\mathcal{B}_{\mathcal{A}} \oplus \mathcal{B}_{\mathcal{B}}$ with Kraus operators $\left\{\operatorname{diag}\left(A_{k}, B_{k}\right)\right\}_{k=1}^{d}$. Obviously, $\mathcal{B}_{\mathcal{A}}$ and $\mathcal{B}_{\mathcal{B}}$ are both BSCCs under $\mathcal{E}$. Then the result follows directly from Lemma 25. 
Note that many interesting results obtained for MPS rely on the basis. For example, one of the most fundamental problems of quantum many-body systems is to identify different tensors that give rise to the same MPS. This problem can be reduced to deciding if the bases of two given tensors are related by a unitary transformation [17]. Thus Theorem 16, which employs simple linear algebra calculation to check if two irreducible tensors are repeated, an essential step in constructing the bases of tensors, can help solve these problems.

\subsection{Conclusion}

In this chapter, with the BSCC and stationary coherence decomposition techniques, we established a structure theory for decoherence-free subsystems. Consequently, a method for finding a set of maximal decoherence-free subsystems has been found. As an application in many-body quantum systems, these results gave us a numerically robust way to find a basis for any tensor by computing the eigenvalues of some linear maps.

For future studies, an immediate topic is to generalize our results to continuoustime quantum systems. In [59], it was studied in the quantum control setting and expected to obtain a linear-algebraic approach for finding all decoherence-free subsystems for any given continuous-time quantum system. 


\section{Chapter 8}

\section{Super-activating Quantum Memory with Entanglement}

Quantum memory is often considered as a method of delayed usage of quantum states and a set of quantum memory cells. The general (hybrid) quantum memory cell was first introduced in [38] and was also termed as noiseless informationpreserving structure [9]. The memory cell has separate (orthogonal) sectors, and each sector is a noiseless subsystem, labeled by a classical "address". Quantum information is stored in each sector with the dimension being at least two, whereas classical information is encoded by the choice between the different sectors. It is reasonable to assume that in a quantum memory, the noise (characterized as a quantum operation) on every memory cell is independent of each other. Moreover, the memory cell is uniquely determined by the noise [9], so we can define a memory cell by a quantum super-operator $\mathcal{E}$. However, useful noiseless subsystems (quantum information can be stored) are limited to the noise that contains some symmetries. Such symmetries are often absent in real devices [64, 63], and thus the memory cell can only preserve classical information if there are at least two sectors; in other words, it is degenerated to be purely classical.

In this chapter, we consider two strategies of using quantum memory cells: individually and collectively. In particular, we observe an interesting new phenomenon: a purely classical memory cell $\mathcal{E}$ can be super-activated to store quantum information; that is, collective use of memory cells can perfectly preserve quantum states while individual memory cell cannot. We find that the memory cell $\mathcal{E}$ can be superactivated if and only if there is an entangled stationary state of $\mathcal{E}^{\otimes 2}$. Furthermore, 
we can give a simple characterization of the super-activation regarding external eigenvalues (with magnitude one) of $\mathcal{E}$. This enables us to easily check the superactivation property as well as the existence of entangled stationary states. Once activated, the maximum dimension of sectors in the memory cell $\mathcal{E}$ may have an exponential growth with the number of used memory cells. Moreover, we see that a null memory cell (no information can be protected) cannot be super-activated to store quantum information, but classical information can be preserved with the collective use of it. This property can be characterized by internal eigenvalues.

This work has been summarized in the paper [24].

\subsection{Quantum Memory}

Given a quantum noise $\mathcal{E}$, recall that noiseless subsystems of $\mathcal{E}$ can be characterized by the set of its fixed points, $\operatorname{fix}(\mathcal{E})=\{A \in \mathcal{L}(\mathcal{H}) \mid \mathcal{E}(A)=A\}$. With an appropriate decomposition of the Hilbert space $\mathcal{H}=\bigoplus_{k=1}^{n}\left(\mathcal{B}_{A_{k}} \otimes \mathcal{B}_{B_{k}}\right) \oplus \mathcal{T}_{\mathcal{E}}$, the fixed points $f i x(\mathcal{E})$ admit the decomposition:

$$
\text { fix }(\mathcal{E})=\bigoplus_{k=1}^{n}\left(\mathcal{L}\left(\mathcal{B}_{A_{k}}\right) \otimes \sigma_{k}\right) \oplus 0_{\mathcal{T}_{\mathcal{E}}}
$$

where $\sigma_{k}$ is a full-rank quantum state on $\mathcal{B}_{B_{k}}$ and $\mathcal{B}_{A_{i}} \otimes \mathcal{B}_{B_{i}}$ is orthogonal to $\mathcal{B}_{A_{j}} \otimes \mathcal{B}_{B_{j}}$ if $i \neq j$.

Using the fixed-point decomposition of a given quantum operation $\mathcal{E}$, we can partition $\eta(\mathcal{E})$, its multiset of eigenvalues with magnitude one, into two parts that play essential roles in the super-activation of quantum memory cells. For each $k$, let $\eta_{k}(\mathcal{E})$ be the multiset of internal eigenvalues of $\mathcal{E}$ restricted on $\mathcal{B}_{A_{k}} \otimes \mathcal{B}_{B_{k}}$ in Eq. 7.2 , again with magnitude one, and let $\bar{\eta}(\mathcal{E})=\eta(\mathcal{E}) \backslash \cup_{k} \eta_{k}(\mathcal{E})$ be the external eigenvalues of $\mathcal{E}$.

To perfectly protect quantum information, a quantum memory cell is defined in [38, 9] as a quantum operation $\mathcal{E}$ to store quantum states into each noiseless 
quantum subsystem $A_{k}$ if the dimension $d_{k}>1$ of $\mathcal{B}_{A_{k}}$. Define the shape of memory cell $\mathcal{E}$ as $\Omega(\mathcal{E})=\left(d_{1}, \cdots, d_{n}\right)$. As the structure of $f i x(\mathcal{E})$ is unique (up to the order of $k), \Omega(\mathcal{E})$ is well-defined. In a sense, $\Omega(\mathcal{E})$ represents the capacity of the memory cell $\mathcal{E}$; that is, how much quantum and classical information can be preserved:

- $|\Omega(\mathcal{E})|_{\infty}=\max _{k} d_{k}$ is the largest dimension of quantum states that can be stored;

- the length $|\Omega(\mathcal{E})|=n$ is the preserved classical information as the choice between the different sectors.

If $|\Omega(\mathcal{E})|_{\infty}=1$ and $|\Omega(\mathcal{E})|>1$, then the quantum memory cell is degenerated to be purely classical, and quantum information cannot be preserved. Furthermore, the memory cell is said to be null if $\Omega(\mathcal{E})=(1)$, i.e. $\mathcal{E}$ is ergodic; that is, neither quantum nor classical information can be stored.

The quantum memory cell model encompasses the existing techniques for preserving quantum and classical information: noiseless subsystems, pointer basis [71] and decoherence-free subspaces [42]. For example, pointer bases have the shape $(1,1, \ldots, 1)$, describing a complete set of one-dimensional $k$ sectors (both $A_{k}$ and $B_{k}$ are trivial for all $k$ ). A decoherence-free subspace has the shape $(d)$, describing a single $k$ sector with a trivial $B_{k}$.

\subsection{Super-activation for Storing Classical Information}

Given a quantum memory cell $\mathcal{E}$, if its shape is $(1, \cdots, 1)$, then quantum information cannot be stored in it. Indeed, it was shown in [64, 63] that in practice only a tiny set of quantum memory cells admits a useful noiseless subsystem (with the dimension being at least 2). Fortunately, this problem can be remedied by the collective use of quantum memory cells where a super-activation of $|\Omega(\cdot)|_{\infty}$ can happen. Let us start with the simplest case with the shape $\Omega(\mathcal{E})=(1)$, i.e., a null 
memory cell. Typical examples include irreducible quantum channels and amplitude damping channels. In this case, memory cell $\mathcal{E}$ behaves periodically in some subspaces.

Lemma 26: Let $\mathcal{E}$ be a quantum memory cell with $\Omega(\mathcal{E})=(1)$. Then there exists some integer $d$ such that

(1) $\eta(\mathcal{E})=\{\exp (2 \pi i k / d)\}_{k=0}^{d-1}$ with each element being internal;

(2) a set of mutually orthogonal states $\left\{\rho_{i}\right\}_{i=0}^{d-1}$ can be found such that $\mathcal{E}\left(\rho_{i}\right)=\rho_{i \boxplus 1}$, where $\boxplus$ denotes addition modulo $d$.

Proof. Please see the Appendix.

It is easy to check that the integer $d(\mathcal{E})=d$ in the above lemma is the period of $\mathcal{E}$. However, note that $d(\mathcal{E})$ is the number of internal eigenvalues. The memory cell $\mathcal{E}$ cannot store quantum and classical information. But we can create new shelters for classical information by using two quantum memory cells with the simplest shapes; that is, $|\Omega(\cdot)|$ can be activated for storing classical information. The following is a simple example of such super-activation.

Example 6: Let $\mathcal{E}$ be a quantum memory cell on $\mathcal{H}=\operatorname{lin} \cdot \operatorname{span}\{|0\rangle,|1\rangle\}$ with $\mathcal{E}(\cdot)=|0\rangle\langle 1|\cdot| 1\rangle\langle 0|+| 1\rangle\langle 0|\cdot| 0\rangle\langle 1|$. It is easy to see that $\Omega(\mathcal{E})=(1)$ and $\Omega\left(\mathcal{E}^{\otimes 2}\right)=$ $(1,1)$, indicating that $|\Omega(\mathcal{E})|$ is activated.

A general characterization of super-activation for storing classical information is presented in the following:

Theorem 17: For any two quantum memory cells $\mathcal{E}$ and $\mathcal{F}$ with $|\Omega(\mathcal{E})|=|\Omega(\mathcal{F})|=$ 1 , there exists a set of mutually orthogonal quantum states $\left\{\rho_{i}\right\}_{i=0}^{m-1}$ with $m=$ $\operatorname{gcd}\{d(\mathcal{E}), d(\mathcal{F})\}$, the greatest common divisor of $d(\mathcal{E})$ and $d(\mathcal{F})$, such that:

$$
f i x(\mathcal{E} \otimes \mathcal{F})=\bigoplus_{i=0}^{m-1} \rho_{i} \oplus 0
$$


that is, $\Omega(\mathcal{E} \otimes \mathcal{F})=(1, \cdots, 1)$ and $|\Omega(\mathcal{E} \otimes \mathcal{F})|=m$. Furthermore, for each $i, \rho_{i}$ is separable.

Proof. As $|\Omega(\mathcal{E})|=|\Omega(\mathcal{F})|=1$, there is only one stationary state $\sigma$ and $\rho$ for $\mathcal{E}$ and $\mathcal{F}$, respectively. By Lemma $26(2)$, there are two sets of mutually orthogonal states $\left\{\sigma_{i}\right\}_{i=0}^{d(\mathcal{E})-1}$ and $\left\{\rho_{i}\right\}_{i=0}^{d(\mathcal{F})-1}$ such that $\mathcal{E}\left(\sigma_{i}\right)=\sigma_{i \boxplus 1}$ and $\mathcal{F}\left(\rho_{i}\right)=\rho_{i \boxplus 1}$. For $0 \leq i \leq m-1$, let

$$
\sigma^{i}=\frac{1}{K_{\mathcal{E}}} \sum_{j=0}^{K_{\mathcal{E}}-1} \sigma_{i \boxplus j m}, \quad \rho^{i}=\frac{1}{K_{\mathcal{F}}} \sum_{j=0}^{K_{\mathcal{F}}-1} \rho_{i \boxplus j m}
$$

where $K_{\mathcal{E}}=d(\mathcal{E}) / m$ and $K_{\mathcal{F}}=d(\mathcal{F}) / m$. We claim that $\left\{\left(\sum_{i} \sigma^{i} \otimes \rho^{i \boxplus j}\right) / m\right\}_{j=0}^{m-1}$ is a set of mutually orthogonal stationary state for $\mathcal{E} \otimes \mathcal{F}$. Indeed, by Lemma 26 (1), we have $\eta(\mathcal{E})=\{\exp (2 \pi i k / d(\mathcal{E}))\}_{k=0}^{d(\mathcal{E})-1}$ and $\eta(\mathcal{F})=\{\exp (2 \pi i k / d(\mathcal{F}))\}_{k=0}^{d(\mathcal{F})-1}$, so the multiplicity of eigenvalue one (for $\mathcal{E} \otimes \mathcal{F}$ ) is $m$. Thus for each $j,\left(\sum_{i=0}^{m-1} \sigma^{i} \otimes \rho^{i \boxplus j}\right) / m$ is a minimal stationary state of $\mathcal{E} \otimes \mathcal{F}$. We finish the proof by noting that it has no other minimal stationary states.

The proof of Theorem 17 gives us an explicit way to construct the memory cell structure, namely the decomposition Eq. (8.1). Usually, entanglement is responsible for the super-activation of many physical quantities in quantum information theory, such as the zero-error capacity of quantum channels [15]. However, the above theorem shows that it is not the case for $|\Omega(\cdot)|$.

For the special case where multiple copies of $\mathcal{E}$ are collectively used, we have:

Corollary 5: Suppose $\mathcal{E}$ is a null memory cell with $|\Omega(\mathcal{E})|=1$. Then

(1) $\left|\Omega\left(\mathcal{E}^{\otimes t}\right)\right|=d(\mathcal{E})^{t-1}$ for any $t \geq 1$. That is, perfect storage of classical information can always be super-activated as long as $d(\mathcal{E})>1$.

(2) $\left|\Omega\left(\mathcal{E}^{\otimes t}\right)\right|_{\infty}=1$ for any $t \geq 1$. That is, no quantum information can be perfectly preserved even collective use of memory cells is employed. 
(3) All stationary states of $\mathcal{E}^{\otimes t}$ are separable.

Proof. By the similar construction of stationary states in the proof of Theorem 17, we obtain $d(\mathcal{E})^{t-1}$ separable and mutually orthogonal stationary states for $\mathcal{E}^{\otimes t}$. Then we compute $\Omega\left(\mathcal{E}^{\otimes t}\right)=(1, \cdots, 1)$ and $\left|\Omega\left(\mathcal{E}^{\otimes t}\right)\right|=d(\mathcal{E})^{t-1}$ by noting that the multiplicity of eigenvalue one (for $\mathcal{E}^{\otimes t}$ ) is $d(\mathcal{E})^{t-1}$.

Note that the period of a null memory cell represents how much classical information can be activated. The above corollary shows that once activation happens, the amount of preserved information can grow up continuously with the number of the application of the memory cells.

\subsection{Super-activation for Storing Quantum Information}

The results presented in the last section show that two null memory cells can be used together to super-activate the amount $|\Omega(\mathcal{E})|$ of stored classical information. In this section, we are going to show how the amount $|\Omega(\mathcal{E})|_{\infty}$ of stored quantum information can be super-activated. The following theorem gives a necessary and sufficient condition for this quantum super-activation.

Theorem 18: Let $\mathcal{E}$ and $\mathcal{F}$ be two quantum memory cells with $|\Omega(\mathcal{E})|_{\infty}=|\Omega(\mathcal{F})|_{\infty}=$ 1. Then the following statements are equivalent:

(1) $|\Omega(\mathcal{E} \otimes \mathcal{F})|_{\infty} \geq 2$

(2) there exists an entangled stationary state for $\mathcal{E} \otimes \mathcal{F}$;

(3) there exist $a \in \eta(\mathcal{E}), b \in \eta(\mathcal{F})$ such that $a b=1$, and $a$ or $b$ is external, i.e. $a \in \bar{\eta}(\mathcal{E})$ or $b \in \bar{\eta}(\mathcal{F})$.

Proof. The implications $(1) \Rightarrow(3)$ and $(2) \Rightarrow(3)$ are from Theorem 17. As $|\Omega(\mathcal{E})|_{\infty}=|\Omega(\mathcal{F})|_{\infty}=1, \Omega(\mathcal{E})=(1, \cdots, 1)$ and $\Omega(\mathcal{F})=(1, \cdots, 1) ;$ that is there are 
only finitely many mutually orthogonal minimal stationary states $\left\{\rho_{i}\right\}$ and $\left\{\sigma_{j}\right\}$ for $\mathcal{E}$ and $\mathcal{F}$, respectively. If for any $a \in \eta(\mathcal{E})$ and $b \in \eta(\mathcal{F}), a b=1$ can only occur when $a$ and $b$ both are internal, i.e. $a \notin \bar{\eta}(\mathcal{E})$ and $b \notin \bar{\eta}(\mathcal{F})$, then it is enough to restrict $\mathcal{E}$ and $\mathcal{F}$ onto the subspaces $\operatorname{supp}\left(\rho_{i}\right)$ and $\operatorname{supp}\left(\sigma_{j}\right)$ respectively, for each $i$ and $j$, when we compute $\operatorname{fix}(\mathcal{E} \otimes \mathcal{F})$. Furthermore, as for each $\rho_{i}$ and $\sigma_{j}$ are minimal, the restricted memory cells $\mathcal{E}_{i}$ and $\mathcal{F}_{j}$ have the simplest shapes. Therefore, following Theorem 17, all stationary states are separable and $|\Omega(\mathcal{E} \otimes \mathcal{F})|_{\infty}=1$, contradicting the assumptions (1) or (2).

To prove $(3) \Rightarrow(1)$ and $(3) \Rightarrow(2)$, let $A$ and $B$ be eigenvectors of $\mathcal{E}$ and $\mathcal{F}$ corresponding to eigenvalues $a$ and $b$ respectively, i.e. $\mathcal{E}(A)=a A$ and $\mathcal{F}(B)=b B$. In the following, we only prove the case that $a \in \bar{\eta}(\mathcal{E})$ and $b \in \bar{\eta}(\mathcal{F})$. Other cases are similar.

From the decomposition Eq. 7.2 of $\mathcal{E}$ and $\mathcal{F}$, there exist mutually orthogonal minimal stationary states $\rho_{1}, \rho_{2}$ for $\mathcal{E}$ and $\sigma_{1}, \sigma_{2}$ for $\mathcal{F}$ such that

$$
\begin{aligned}
& A \in \operatorname{lin} . \operatorname{span}\left\{\left|\psi_{1}\right\rangle\left\langle\psi_{2}|:| \psi_{i}\right\rangle \in \operatorname{supp}\left(\rho_{i}\right), i=1,2\right\} \\
& B \in \operatorname{lin} . \operatorname{span}\left\{\left|\phi_{1}\right\rangle\left\langle\phi_{2}|:| \phi_{i}\right\rangle \in \operatorname{supp}\left(\sigma_{i}\right), i=1,2\right\}
\end{aligned}
$$

Then we can find a positive number $\epsilon$ such that

$$
\frac{1}{K}\left[\rho_{1} \otimes \sigma_{1}+\rho_{2} \otimes \sigma_{2}+\epsilon\left(A \otimes B+A^{\dagger} \otimes B^{\dagger}\right)\right]
$$

is a stationary state for $\mathcal{E} \otimes \mathcal{F}$, where $K$ is a normalization factor. Note that this state is entangled by the positive partial transpose criteria [48], thus (2) holds.

Suppose $|\Omega(\mathcal{E} \otimes \mathcal{F})|_{\infty}=1$. Then there are finitely many mutually orthogonal minimal stationary states $\left\{\xi_{i}\right\}_{i=1}^{m}$ for $\mathcal{E} \otimes \mathcal{F}$. By [66, Corollary 6.5], it contradicts the fact that $A \otimes B, \rho_{1} \otimes \sigma_{1}$ and $\rho_{2} \otimes \sigma_{2}$ can all be linearly represented by $\left\{\xi_{i}\right\}_{i=1}^{m}$. This proves (1). 
Corollary 6: Given a quantum memory cell $\mathcal{E},|\Omega(\mathcal{E})|_{\infty}$ can be super-activated if and only if the multiset $\bar{\eta}(\mathcal{E})$ of external eigenvalues is not empty. Furthermore, $\left|\Omega\left(\mathcal{E}^{\otimes t}\right)\right|_{\infty}$ is increasing with $t$.

Proof. It suffices to note that the set of eigenvalues of $\mathcal{E}$ is closed under complex conjugate. Furthermore, if $n>m$, the noiseless subsystems of $\mathcal{E}^{\otimes m}$ is also the noiseless subsystems of $\mathcal{E}^{\otimes n}$, so $\left|\Omega\left(\mathcal{E}^{\otimes n}\right)\right|_{\infty} \geq\left|\Omega\left(\mathcal{E}^{\otimes m}\right)\right|_{\infty}$.

Note that computing $\bar{\eta}(\mathcal{E})$ is an easy linear algebra exercise. Thus super-activation of a given quantum memory cell $\mathcal{E}$ can be checked easily without finding an entangled stationary state or the noiseless subsystems of $\mathcal{E}^{\otimes t}$.

Theorem 18 and Corollary 6 have some interesting implications. First, if we want to super-activate $|\Omega(\mathcal{E})|_{\infty}$ by collective use of $\mathcal{E}, \mathcal{E}$ must have at least two (mutually orthogonal) stationary quantum states; i.e. $|\Omega(\mathcal{E})|>1$. This means that classical information can be stored in the memory cell $\mathcal{E}$. Therefore, such superactivation implies the preservation of classical information. This is in sharp contrast to the super-activation in zero-error communication over quantum channels: there exist quantum channels $\mathcal{F}_{1}$ and $\mathcal{F}_{2}$ such that both of them have vanishing zero-error classical capacity (meaning that classical information cannot be sent without errors), but the zero-error quantum capacity of $\mathcal{F}_{1} \otimes \mathcal{F}_{2}$ is positive (meaning that we can use it to transmit quantum information perfectly) [16]. Secondly, if an entangled stationary state is found, then there is at least one useful noiseless subsystem in the whole memory cell system that can be used to store (entangled) quantum states. So, entanglement can be served as a signal for protecting quantum information, like the period in the super-activation of $|\Omega(\cdot)|$. Thirdly, the quantities $|\Omega(\cdot)|_{\infty}$ and $|\Omega(\cdot)|$ are not multiplicative, i.e. in general $|\Omega(\mathcal{E} \otimes \mathcal{F})|_{\infty} \neq|\Omega(\mathcal{E})|_{\infty} \cdot|\Omega(\mathcal{F})|_{\infty}$ and $|\Omega(\mathcal{E} \otimes \mathcal{F})| \neq|\Omega(\mathcal{E})| \cdot|\Omega(\mathcal{F})|$. This implies that the amount of information that can be preserved through a quantum memory cell depends on what other memory cells 
are also available.

Mathematically, given a quantum memory cell $\mathcal{E}$, the shape is entirely determined by its magnitude 1-eigenvalues and the corresponding eigenvectors, and the superactivation of $|\Omega(\cdot)|$ and $|\Omega(\mathcal{E})|_{\infty}$ is determined by internal and external eigenvalues of $\mathcal{E}$, respectively. This indicates that eigenvalues with magnitude one have different roles in information storage.

Finally, we present a simple example to show that the growth of the superactivation of $|\Omega(\cdot)|_{\infty}$ can be exponentially fast, and the speed of growth is independent on external eigenvalues. Therefore, the collective use of purely classical memory cells is an efficient method to preserve quantum information.

Example 7: Let $\theta_{1}, \theta_{2}$ be real numbers and $0<\theta_{1} \leq \theta_{2}<2 \pi$. We consider two quantum memory cells $\mathcal{E}_{k}(\rho)=\left(|0\rangle\left\langle 0\left|+e^{i \theta_{k}}\right| 1\right\rangle\langle 1|\right) \rho\left(|0\rangle\left\langle 0\left|+e^{-i \theta_{k}}\right| 1\right\rangle\langle 1|\right)+$ $|2\rangle\langle 2|\rho| 2\rangle\langle 2|$ on $\mathcal{H}=$ lin.span $\{|0\rangle,|1\rangle,|2\rangle\}$ for $k=1,2$. Note that for each $k$, $\mathcal{E}_{k}(\rho)=\left(|0\rangle\left\langle 0\left|+e^{i \theta_{k}}\right| 1\right\rangle\langle 1|\right)\left(P_{0}+P_{1}\right) \rho\left(P_{0}+P_{1}\right)\left(|0\rangle\left\langle 0\left|+e^{-i \theta_{k}}\right| 1\right\rangle\langle 1|\right)+|2\rangle\left\langle 2\left|P_{2} \rho P_{2}\right| 2\right\rangle\langle 2|$, where $P_{j}$ is the projection onto lin.span $\{|j\rangle\}$ for $j \in\{0,1,2\}$. So, we can restrict $\mathcal{E}_{k}$ onto lin.span $\{|0\rangle,|1\rangle\}$ when we only consider $|\Omega(\cdot)|_{\infty}$. Then the evolution is fully represented by unitary matrices $\left\{U_{k}=\operatorname{diag}\left(1, e^{i \theta_{k}}\right)\right\}_{k=1,2}$. It is easy to compute $\Omega\left(\mathcal{E}_{k}\right)=(1,1,1)$, and $\bar{\eta}\left(\mathcal{E}_{k}\right)=\left\{e^{i \theta_{k}}, e^{-i \theta_{k}}\right\}$ for $k=1,2$. By Theorem 18 , $\left|\Omega\left(\mathcal{E}_{1} \otimes \mathcal{E}_{2}\right)\right|_{\infty}>1$ if and only if $\theta_{1}=\theta_{2}$ or $\theta_{1}=2 \pi-\theta_{2}$. Now, we show that the growth speed of the super-activation is independent on $\theta_{1}, \theta_{2}$ and exponential with the number of collectively used memory cells in both cases.

(1) $\theta_{1}=\theta_{2}$. Then let $U=U_{1}=U_{2}$ and $\mathcal{E}=\mathcal{E}_{1}=\mathcal{E}_{2}$. For any strictly positive integer $t$,

$$
U^{\otimes t} \simeq \operatorname{diag}\left[I_{\left(\begin{array}{c}
t \\
0
\end{array}\right)}, I_{\left(\begin{array}{l}
t \\
1
\end{array}\right)} e^{i \theta}, \cdots, I_{\left(\begin{array}{c}
t \\
t
\end{array}\right)} e^{i t \theta}\right]
$$

where $I_{k}$ is the identity matrix with dimension $k$. Then $\left|\Omega\left(\mathcal{E}^{\otimes t}\right)\right|_{\infty}>\left(\begin{array}{c}t \\ t / 2\end{array}\right)$ if $t$ is even; otherwise, $\left|\Omega\left(\mathcal{E}^{\otimes t}\right)\right|_{\infty}>\left(\begin{array}{c}t \\ (t-1) / 2\end{array}\right)$. 
(2) $\theta_{1}=2 \pi-\theta_{2}$, i.e. $U_{1}=U_{2}^{\dagger}$. For any strictly positive integer $t, \mid \Omega\left[\left(\mathcal{E}_{1} \otimes\right.\right.$ $\left.\left.\mathcal{E}_{2}\right)^{\otimes t}\right]\left.\right|_{\infty} \geq\left(\begin{array}{c}t \\ t / 2\end{array}\right)^{2}$ if $t$ is even; otherwise $\left|\Omega\left[\left(\mathcal{E}_{1} \otimes \mathcal{E}_{2}\right)^{\otimes t}\right]\right|_{\infty} \geq\left(\begin{array}{c}t \\ (t-1) / 2\end{array}\right)^{2}$.

Therefore, in the above cases, the growth speed is independent on $\theta_{1}, \theta_{2}$. Specifically, by Stirling's approximation, $\left(\begin{array}{c}t \\ t / 2\end{array}\right)$ and $\left(\begin{array}{c}t \\ (t-1) / 2\end{array}\right)$ both are growing up exponentially with $t$ and $\left|\Omega\left(\mathcal{E}^{\otimes t}\right)\right|_{\infty} \leq n^{t}$ with $\operatorname{dim}(\mathcal{H})=n$, so $|\Omega(\cdot)|_{\infty}$ has an exponential growth.

\subsection{Conclusion}

Using the stationary coherence and periodic decompositions, we proved that the existence of entangled stationary states of a given purely classical memory cell is necessary and sufficient for super-activating it to store quantum information, whereas a null memory cell can only be super-activated to preserve classical information. We also proposed a simple method to check whether such super-activation happens by computing its external and internal eigenvalues, respectively. Moreover, once activated, the preserved quantum information may have exponential growth with the number of the used memory cells. This provides an efficient way to perfectly preserve quantum information even when the quantum memory cell is fully classical.

At this moment, we only have a simple example showing the exponential growth of super-activation of the amount $|\Omega(\cdot)|_{\infty}$ of stored quantum information. In future research, we expect to give a general characterization of the growth speed of $|\Omega(\cdot)|_{\infty}$. 


\section{Part V}

Concluding Remarks 
In this thesis, we presented a new decomposition of quantum Markov chains, namely periodic decomposition. This decomposition helped us build a limit theorem for (irreducible) quantum Markov chains. More importantly, we applied it with the formerly developed decompositions (the BSCC and stationary coherence decompositions) in quantum information theory, especially protecting quantum states, and got three significant results:

- A novel characterization of the one-shot zero-error capacity of quantum channels;

- The structure of quantum decoherence-free subsystems;

- Entanglement super-activating quantum memory.

For further studies, there are several potential fields where the three-level decomposition technique can be used:

- Reachability analysis of quantum Markov chains: The BSCC decomposition was already used in reachability analysis of quantum Markov chains [68]. The eventual, global, ultimately forever and infinitely often reachability of quantum automata were carefully examined in [41]. Quantum automata is a special kind of quantum Markov chains, where the dynamics is described by a unitary transformation rather than a general super-operator. It seems that the threelevel decomposition presented in this thesis is useful for analysis of these more sophisticated reachabilities of quantum Markov chains.

- Extend the decomposition techniques developed in this thesis to quantum Markov decision processes, which were introduced in [4] for quantum machine learning and in 69] for modeling concurrent quantum programs. 


\section{Part VI}

Appendix 


\section{Proof of Lemma 26}

Lemma 2\%: Let $\mathcal{E}$ be a quantum operation on $\mathcal{H}$ with the fixed-point decomposition $\mathcal{H}=\bigoplus_{k=1}^{n}\left(\mathcal{B}_{A_{k}} \otimes \mathcal{B}_{B_{k}}\right) \oplus \mathcal{T}_{\mathcal{E}}$, and $X$ an eigenvector corresponding to some $a \in \eta(\mathcal{E})$. Then $X \in \mathcal{L}\left(\mathcal{T}_{\mathcal{E}}^{\perp}\right)$.

Proof. By the definition, we first observe that $\mathcal{E}_{\phi}(X)=X$ for all $X$ with $\mathcal{E}(X)=$ $a X$ for some $a \in \eta(\mathcal{E})$. Recall that $\mathcal{T}_{\mathcal{E}}$ is transitive, i.e. for all $\rho \in D(\mathcal{H})$, $\lim _{n \rightarrow \infty} \operatorname{tr}\left(P \mathcal{E}^{n}(\rho)\right)=0$, where $P$ is the projection onto $\mathcal{T}_{\mathcal{E}}$. Furthermore, Lemma 3 asserts that there exists an increasing sequence of integers $n_{i}$ such that $\mathcal{E}_{\phi}=$ $\lim _{i \rightarrow \infty} \mathcal{E}^{n_{i}}$. Therefore, $\operatorname{tr}\left(P \mathcal{E}_{\phi}(\rho)\right)=0$ for all $\rho \in D(\mathcal{H})$. The lemma follows by noting the structure of $f i x\left(\mathcal{E}_{\phi}\right)$ and that $X$ can be linearly represented by a set of quantum states.

If we only want to compute eigenvalues of $\mathcal{E}$ with magnitude one and corresponding eigenvectors, then the restriction of $\mathcal{E}$ onto $\mathcal{T}_{\mathcal{E}}^{\perp}$ is enough to be used by lemma 27. Furthermore, the following lemma gives a characterization of these eigenvalues when $\Omega(\mathcal{E})=(1)$; i.e. $\mathcal{E}$ is ergodic.

Lemma 28: For any quantum operation $\mathcal{E}$ with $\Omega(\mathcal{E})=(1)$, we have $\eta(\mathcal{E})=$ $\{\exp (2 \pi i k / d)\}_{k=0}^{d-1}$ for some integer $d$, and all elements in $\eta(\mathcal{E})$ are internal.

Proof. As $\Omega(\mathcal{E})=(1)$, there is only one stationary state $\rho^{*}$ of $\mathcal{E}$. By restricting $\mathcal{E}$ onto $\mathcal{X}=\operatorname{supp}\left(\rho^{*}\right), \mathcal{E}_{\mathcal{X}}$ is irreducible; that is, its shape is (1) and its stationary state is of full-rank. Then with [66, Theorem 6.6], $\eta(\mathcal{E}) \supseteq \eta\left(\mathcal{E}_{\mathcal{X}}\right)=\{\exp (2 \pi i k / d)\}_{k=0}^{d-1}$ for some integer $d$, and the multiplicity of any eigenvalue in $\eta\left(\mathcal{E}_{\mathcal{X}}\right)$ is 1 . Following Lemma 27, there are no other eigenvalues in $\eta(\mathcal{E})$, so $\eta(\mathcal{E})=\eta\left(\mathcal{E}_{\mathcal{X}}\right)$.

Corollary 7: Given a quantum operation $\mathcal{E}$, if $\Omega(\mathcal{E})=(m)$ for some positive integer $m$, then $\eta(\mathcal{E})=\{\exp (2 \pi i k / d)\}_{k=0}^{d-1}$ for some integer $d$, and all elements in $\eta(\mathcal{E})$ have multiplicity $m$. 
For a quantum operation $\mathcal{E}$ with $\Omega(\mathcal{E})=(1)$, i.e. $\mathcal{E}$ is ergodic, we can claim that the period of $\mathcal{E}$ to be the number $d$ by the above lemma. We can further get a more interesting result:

Lemma 29: For a quantum operation $\mathcal{E}$ with $\Omega(\mathcal{E})=(1)$, there exists a set of mutually orthogonal quantum states $\left\{\rho_{i}\right\}_{i=0}^{d(\mathcal{E})-1}$ such that $\mathcal{E}\left(\rho_{i}\right)=\rho_{i \boxplus 1}$, where $\boxplus$ denotes addition modulo $d(\mathcal{E})$.

Proof. Without loss of generality, we assume that $\mathcal{E}$ is irreducible. Otherwise, we restrict $\mathcal{E}$ onto the support of the stationary state. Let $d=d(\mathcal{E})$. By Theorem 8 , there exists a set of mutually orthogonal subspaces $\left\{\mathcal{B}_{i}\right\}_{i=0}^{d-1}$ such that $\mathcal{H}=\bigoplus_{i} \mathcal{B}_{i}$ and for each $i, \mathcal{B}_{i}$ is invariant under $\mathcal{E}^{d}$. Then for each $i,\left.\mathcal{E}^{d}\right|_{\mathcal{B}_{i}}$, the restriction of $\mathcal{E}^{d}$ onto $\mathcal{B}_{i}$, has a limit state, i.e. there is a quantum state $\sigma_{i} \in D\left(\mathcal{B}_{i}\right)$ such that for all $\rho \in D\left(\mathcal{B}_{i}\right),\left.\lim _{n \rightarrow \infty} \mathcal{E}^{d n}\right|_{\mathcal{B}_{i}}(\rho)=\sigma_{i}$. As $\mathcal{B}_{i}$ is an invariant subspace of $\mathcal{E}^{d}$, this means $\lim _{n \rightarrow \infty} \mathcal{E}^{d n}(\rho)=\sigma_{i}$. From [25, Theorem 7], we have $\mathcal{E}(\rho) \in D\left(\mathcal{B}_{i \boxplus 1}\right)$. So,

$$
\begin{aligned}
\lim _{n \rightarrow \infty} \mathcal{E}^{d n}(\rho)=\sigma_{i} & \Rightarrow \quad \lim _{n \rightarrow \infty} \mathcal{E}^{d n+1}(\rho)=\mathcal{E}\left(\sigma_{i}\right) \\
& \Rightarrow \quad \lim _{n \rightarrow \infty} \mathcal{E}^{d n}(\mathcal{E}(\rho))=\mathcal{E}\left(\sigma_{i}\right) \\
& \left.\Rightarrow \quad \lim _{n \rightarrow \infty} \mathcal{E}^{d n}\right|_{\mathcal{B}_{i \boxplus 1}}(\mathcal{E}(\rho))=\mathcal{E}\left(\sigma_{i}\right) \\
& \Rightarrow \quad \sigma_{i \boxplus 1}=\mathcal{E}\left(\sigma_{i}\right) .
\end{aligned}
$$




\section{Bibliography}

[1] D. Acemoglu, G. Egorov, and K. Sonin, "Political model of social evolution," Proceedings of the National Academy of Sciences, vol. 108, no. Supplement 4, pp. $21292-21296,2011$.

[2] A. Ambainis, "Quantum walks and their algorithmic applications," International Journal of Quantum Information, vol. 1, no. 04, pp. 507-518, 2003.

[3] C. Baier, J.-P. Katoen, and K. G. Larsen, Principles of model checking. MIT press, 2008.

[4] J. Barry, D. T. Barry, and S. Aaronson, "Quantum partially observable markov decision processes," Physical Review A, vol. 90, no. 3, p. 032311, 2014.

[5] B. Baumgartner and H. Narnhofer, "The structures of state space concerning quantum dynamical semigroups," Reviews in Mathematical Physics, vol. 24, no. 02 , p. $1250001,2012$.

[6] S. Beigi and P. W. Shor, "On the complexity of computing zero-error and holevo capacity of quantum channels," arXiv preprint arXiv:0709.2090, 2007.

[7] C. Bény, A. Kempf, and D. W. Kribs, "Generalization of quantum error correction via the heisenberg picture," Physical review letters, vol. 98, no. 10, p. $100502,2007$.

[8] R. Blume-Kohout, H. K. Ng, D. Poulin, and L. Viola, "Characterizing the structure of preserved information in quantum processes," Physical review letters, vol. 100, no. 3, p. 030501, 2008. 
[9] — , "Information-preserving structures: A general framework for quantum zero-error information," Physical Review A, vol. 82, no. 6, p. 062306, 2010.

[10] D. Burgarth, G. Chiribella, V. Giovannetti, P. Perinotti, and K. Yuasa, "Ergodic and mixing quantum channels in finite dimensions," New Journal of Physics, vol. 15, no. 7, p. 073045, 2013.

[11] R. Carbone and Y. Pautrat, "Open quantum random walks: reducibility, period, ergodic properties," in Annales Henri Poincaré, vol. 17, no. 1. Springer, 2016, pp. 99-135.

[12] J. Chen, T. S. Cubitt, A. W. Harrow, and G. Smith, "Entanglement can completely defeat quantum noise," Physical review letters, vol. 107, no. 25, p. $250504,2011$.

[13] M.-D. Choi and D. W. Kribs, "Method to find quantum noiseless subsystems," Physical review letters, vol. 96, no. 5, p. 050501, 2006.

[14] J. I. Cirac, D. Perez-Garcia, N. Schuch, and F. Verstraete, "Matrix product density operators: Renormalization fixed points and boundary theories," $A n$ nals of Physics, vol. 378, pp. 100-149, 2017.

[15] T. S. Cubitt, J. Chen, and A. W. Harrow, "Superactivation of the asymptotic zero-error classical capacity of a quantum channel," IEEE transactions on information theory, vol. 57, no. 12, pp. 8114-8126, 2011.

[16] T. S. Cubitt and G. Smith, "An extreme form of superactivation for quantum zero-error capacities," IEEE Transactions on Information Theory, vol. 58, no. 3, pp. 1953-1961, 2012.

[17] G. D. 1. Cuevas, J. I. Cirac, N. Schuch, and D. Perez-Garcia, "Irreducible forms of matrix product states: Theory and applications," arXiv preprint arXiv:1708.00029, 2017. 
[18] E. Davies, "Quantum stochastic processes," Communications in Mathematical Physics, vol. 15, no. 4, pp. 277-304, 1969.

[19] — - "Quantum stochastic processes ii," Communications in Mathematical Physics, vol. 19, no. 2, pp. 83-105, 1970.

[20] R. Duan, S. Severini, and A. Winter, "Zero-error communication via quantum channels, noncommutative graphs, and a quantum lovász number," IEEE Transactions on Information Theory, vol. 59, no. 2, pp. 1164-1174, 2013.

[21] F. Fagnola and R. Pellicer, "Irreducible and periodic positive maps," Commun. Stoch. Anal, vol. 3, no. 3, pp. 407-418, 2009.

[22] F. Fagnola and R. Rebolledo, "On the existence of stationary states for quantum dynamical semigroups," Journal of Mathematical Physics, vol. 42, no. 3, pp. 1296-1308, 2001.

[23] P. A. Gagniuc, Markov Chains: From Theory to Implementation and Experimentation. John Wiley \& Sons, 2017.

[24] J. Guan, Y. Feng, and M. Ying, "Super-activating quantum memory with entanglement," arXiv preprint arXiv:1708.00700, 2017.

[25] — - "Decomposition of quantum markov chains and its applications," Journal of Computer and System Sciences, vol. 95, pp. 55-68, 2018.

[26] —, "The structure of decoherence-free subsystems," arXiv preprint arXiv:1802.04904, 2018.

[27] O. Häggström, Finite Markov chains and algorithmic applications. Cambridge University Press, 2002, vol. 52. 
[28] J. D. Hamilton, "A new approach to the economic analysis of nonstationary time series and the business cycle," Econometrica: Journal of the Econometric Society, pp. 357-384, 1989.

[29] R. A. Horn and C. R. Johnson, Matrix analysis. Cambridge university press, 2012 .

[30] P. D. Johnson, F. Ticozzi, and L. Viola, "General fixed points of quasi-local frustration-free quantum semigroups: from invariance to stabilization," arXiv preprint arXiv:1506.07756, 2015.

[31] J. Kempe, "Quantum random walks: an introductory overview," Contemporary Physics, vol. 44, no. 4, pp. 307-327, 2003.

[32] A. Kitaev and M. N. Shen, A. H.and Vyalyi, Classical and Quantum Computation. American Mathematical Society, 2002.

[33] E. Knill, "Protected realizations of quantum information," Physical Review A, vol. 74, no. 4, p. 042301, 2006.

[34] E. Knill, R. Laflamme, and L. Viola, "Theory of quantum error correction for general noise," Physical Review Letters, vol. 84, no. 11, p. 2525, 2000.

[35] D. Kribs, R. Laflamme, and D. Poulin, "Unified and generalized approach to quantum error correction," Physical review letters, vol. 94, no. 18, p. 180501, 2005 .

[36] D. W. Kribs, R. Laflamme, D. Poulin, and M. Lesosky, "Operator quantum error correction," arXiv preprint quant-ph/0504189, 2005.

[37] D. W. Kribs and R. W. Spekkens, "Quantum error-correcting subsystems are unitarily recoverable subsystems," Physical Review A, vol. 74, no. 4, p. 042329, 2006. 
[38] G. Kuperberg, "The capacity of hybrid quantum memory," IEEE Transactions on Information Theory, vol. 49, no. 6, pp. 1465-1473, 2003.

[39] P. S. Kutchukian, D. Lou, and E. I. Shakhnovich, "Fog: Fragment optimized growth algorithm for the de novo generation of molecules occupying druglike chemical space," Journal of chemical information and modeling, vol. 49, no. 7, pp. 1630-1642, 2009.

[40] D. A. Levin, Y. Peres, and E. L. Wilmer, Markov chains and mixing times. American Mathematical Soc., 2009.

[41] Y. Li and M. Ying, "(un) decidable problems about reachability of quantum systems," in International Conference on Concurrency Theory. Springer, 2014, pp. $482-496$.

[42] D. A. Lidar, "Review of decoherence free subspaces, noiseless subsystems, and dynamical decoupling," arXiv preprint arXiv:1208.5791, 2012.

[43] D. A. Lidar, I. L. Chuang, and K. B. Whaley, "Decoherence-free subspaces for quantum computation," Physical Review Letters, vol. 81, no. 12, p. 2594, 1998.

[44] G. Lindblad, "On the generators of quantum dynamical semigroups," Communications in Mathematical Physics, vol. 48, no. 2, pp. 119-130, 1976.

[45] C. Liu and N. Petulante, "On limiting distributions of quantum markov chains," International Journal of Mathematics and Mathematical Sciences, vol. 2011, 2011.

[46] K. McAlpine, E. Miranda, and S. Hoggar, "Making music with algorithms: A case-study system," Computer Music Journal, vol. 23, no. 2, pp. 19-30, 1999.

[47] R. A. Medeiros, R. Alléaume, G. Cohen, and F. M. de Assis, "Zero-error capacity of quantum channels and noiseless subsystems," in Telecommunications 
Symposium, 2006 International. IEEE, 2006, pp. 900-905.

[48] F. Mintert, C. Viviescas, and A. Buchleitner, "Basic concepts of entangled states," in Entanglement and Decoherence. Springer, 2009, pp. 61-86.

[49] M. A. Nielsen and I. L. Chuang, Quantum computation and quantum information. Cambridge university press, 2010.

[50] L. Page, "Method for node ranking in a linked database," Sep. 4 2001, uS Patent 6,285,999.

[51] V. Paulsen, Completely bounded maps and operator algebras. Cambridge University Press, 2002, vol. 78 .

[52] D. Pratas, R. M. Silva, A. J. Pinho, and P. J. Ferreira, "An alignment-free method to find and visualise rearrangements between pairs of dna sequences," Scientific reports, vol. 5, 2015.

[53] M. Sanz, D. Pérez-García, M. M. Wolf, and J. I. Cirac, "A quantum version of wielandt's inequality," IEEE Transactions on Information Theory, vol. 56, no. 9, pp. 4668-4673, 2010.

[54] P. Scaramuzza and F. Ticozzi, "Switching quantum dynamics for fast preparation of pure states," in Decision and Control (CDC), 2015 IEEE 54th Annual Conference on. IEEE, 2015, pp. 6434-6440.

[55] S. Schirmer, A. Solomon, and J. Leahy, "Criteria for reachability of quantum states," Journal of Physics A: Mathematical and General, vol. 35, no. 40, p. 8551, 2002.

[56] A. Shabani and D. A. Lidar, "Theory of initialization-free decoherence-free subspaces and subsystems," Physical Review A, vol. 72, no. 4, p. 042303, 2005. 
[57] C. Shannon, "The zero error capacity of a noisy channel," IRE Transactions on Information Theory, vol. 2, no. 3, pp. 8-19, 1956.

[58] F. Ticozzi, S. G. Schirmer, and X. Wang, "Stabilizing quantum states by constructive design of open quantum dynamics," IEEE Transactions on Automatic Control, vol. 55, no. 12, pp. 2901-2905, 2010.

[59] F. Ticozzi and L. Viola, "Quantum markovian subsystems: invariance, attractivity, and control," IEEE Transactions on Automatic Control, vol. 53, no. 9, pp. 2048-2063, 2008.

[60] —_ "Analysis and synthesis of attractive quantum markovian dynamics," Automatica, vol. 45, no. 9, pp. 2002-2009, 2009.

[61] — , "Steady-state entanglement by engineered quasi-local markovian dissipation," arXiv preprint arXiv:1304.4270, 2013.

[62] V. Umanità, "Classification and decomposition of quantum markov semigroups," Probability theory and related fields, vol. 134, no. 4, pp. 603-623, 2006.

[63] X. Wang, M. Byrd, and K. Jacobs, "Numerical method for finding decoherencefree subspaces and its applications," Physical Review A, vol. 87, no. 1, p. 012338, 2013.

[64] — - "Minimal noise subsystems," Physical review letters, vol. 116, no. 9, p. 090404, 2016.

[65] H. Wielandt, "Unzerlegbare, nicht negative matrizen," Mathematische Zeitschrift, vol. 52, no. 1, pp. 642-648, 1950.

[66] M. M. Wolf, "Quantum channels \& operations: Guided tour," Lecture notes available at http://www-m5. ma. tum. de/foswiki/pub M, vol. 5, 2012. 
[67] M. Ying, N. Yu, Y. Feng, and R. Duan, "Verification of quantum programs," Science of Computer Programming, vol. 78, pp. 1679-1700, 2013.

[68] S. Ying, Y. Feng, N. Yu, and M. Ying, "Reachability probabilities of quantum markov chains," in International Conference on Concurrency Theory. Springer, 2013, pp. 334-348.

[69] S. Ying and M. Ying, "Reachability analysis of quantum markov decision processes," arXiv preprint arXiv:1406.6146, 2014.

[70] N. Yu and M. Ying, "Reachability and termination analysis of concurrent quantum programs," in International Conference on Concurrency Theory. Springer, 2012, pp. 69-83.

[71] W. H. Zurek, "Pointer basis of quantum apparatus: Into what mixture does the wave packet collapse?" Physical Review D, vol. 24, no. 6, p. 1516, 1981. 